PAPER • OPEN ACCESS

\title{
Universal break law for a class of models of polymer rupture
}

To cite this article: Frank Aurzada et al 2021 J. Phys. A: Math. Theor. 54305204

View the article online for updates and enhancements.

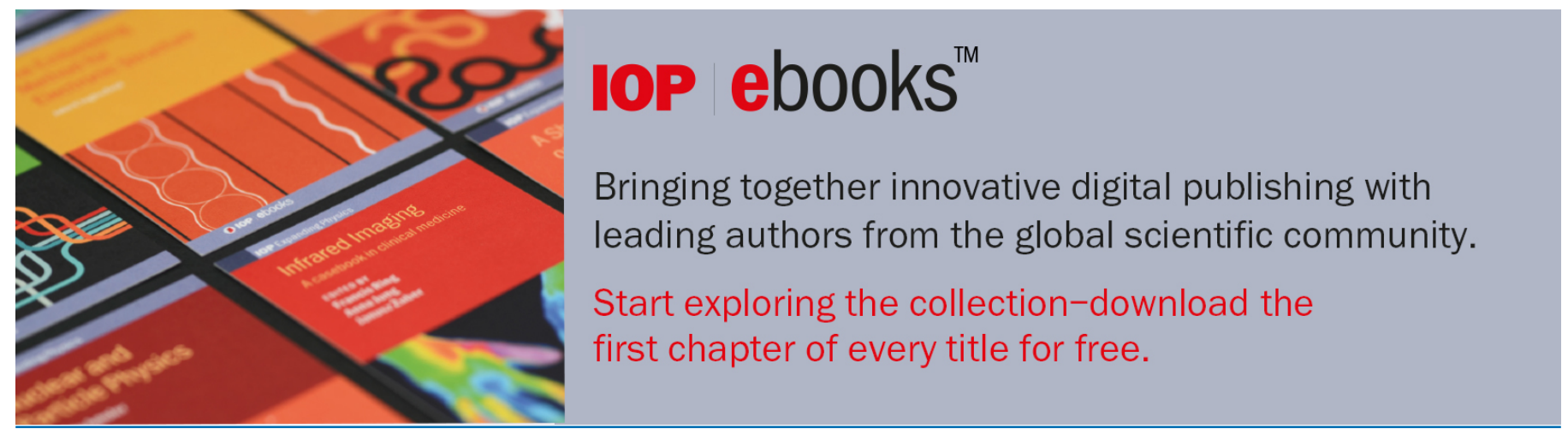




\title{
Universal break law for a class of models of polymer rupture
}

\author{
Frank Aurzada ${ }^{1, *}\left(\mathbb{D}\right.$, Volker Betz $^{1, *}(\mathbb{D})$ and Mikhail Lifshits ${ }^{2, *}$ \\ 1 Technische Universität Darmstadt, Germany \\ 2 St. Petersburg State University, Russia \\ E-mail: aurzada@mathematik.tu-darmstadt.de,betz@mathematik.tu-darmstadt.de \\ and mikhail@lifshits.org
}

Received 6 April 2021, revised 5 June 2021

Accepted for publication 16 June 2021

Published 2 July 2021

\begin{abstract}
We model a polymer by a finite chain of Brownian particles, interacting through a pairwise potential $U$. We investigate what happens when one end of the chain is fixed and the other end slowly pulled away, and when we assume that the chain breaks as soon as the distance between two neighbouring particles exceeds a certain threshold $b$. We find that under natural conditions on $U$ and suitable scaling of noise and pulling speed, the laws of the break time and of the place along the chain where the break occurs converge to explicit limits. These limits are universal in the sense that they only depend on $U^{\prime \prime}(b)$.
\end{abstract}

Keywords: interacting Brownian particles, rupture of a molecular chain, stochastic differential equations

\section{Introduction}

The rupture of polymer chains under strain has been an active research topic for many years. A natural model is to regard the chain as a string of particles coupled by a nearest neighbour force and subjected to thermal fluctuations. An early account of this point of view can be found in [8], where also a good survey of older and different approaches is contained. While in [8], the chain is modelled by Newtonian dynamics, recently the more tractable model of overdamped particles has received more attention. In $[9,10]$, one end of an overdamped chain in a thermal environment is anchored at a fixed position, while the other end is pulled with a force that is increasing linearly in time. One is interested in the (random) force (equivalently, the random time) where the chain breaks, as a function of the length $N$ of the chain.

*Authors to whom any correspondence should be addressed.

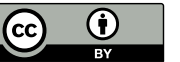

Original content from this work may be used under the terms of the Creative Commons Attribution 4.0 licence. Any further distribution of this work must maintain attribution to the author(s) and the title of the work, journal citation and DOI. 
It is found that the average force necessary for breaking the chain is first decreasing with $N$, but then starts increasing for even larger $N$ before saturating in the limit of very long chains.

A slightly different scenario is when the chain is elongated by a force that does not depend on time, but is reasonably close to the force necessary to break the chain without influence of thermal fluctuations. In this setting [], study the dependence of the breaking rates on the chosen potential, and it is found that the Lennard-Jones and Morse potentials lead to different rupture rates at equal barrier height. In $[12,19]$, the question is asked at which point along the chain a rupture occurs - in [12] it is found that in an elongated chain in the limit of small noise, the rupture location is uniform along the chain, with the exception of the two extremal bonds where the rate is twice as high as at the other bonds [19] goes beyond the limit of small noise and finds that for finite noise, the middle bonds are somewhat less likely to break than the ones close to the boundary. In [], for a Morse type potential a power law tail of the distribution of break rates is discovered, which is universal in the sense that it does not depend on the details of the potential.

All of the above papers work on the physical level of rigour and in the adiabatic setting, i.e. they assume that at each point in time the distribution of the particles in the chain is stationary given the force applied at that point in time. They then use various expansions and approximations to reach their conclusions and sometimes validate their findings using molecular dynamics simulations. A more fundamental type of programme has been started in [1] There, the chain is modelled as a string of Brownian particles interacting via a nearest neighbour potential (in the sense of particle labels). The left end of the chain is anchored, but the right end is pulled at constant speed, leading to a situation that is most closely related to the one studied in $[9,10]$ where the chain is pulled with a force increasing in time. Rupture is defined in terms of a first passage time of the paths of the resulting stochastic process: as soon as the distance between two neighbouring particles exceeds a certain threshold, the chain is declared to be broken. The potential is assumed to be convex up to that threshold, in contrast to the situation in most of the above cited references, where the break occurs at the inflection point of the Morse potential.

Under these assumptions [1], finds a mathematically rigorous theory of rupture in various regimes of noise strength in relation to elongation speed. However, an important restriction in that paper was that the chain had to consist of only three particles, meaning in particular that only one particle was able to move non-deterministically. This restriction was overcome recently in [3, 4], where chains of arbitrary length are considered, however at the expense of another restriction: in these papers, the inter-particle potential is assumed to be quadratic, and thus the first passage probabilities can be estimated using the very detailed large deviation estimates available for Gaussian processes. The present paper completes the programme started in [1] by removing the restriction to Gaussian processes in the most important regime, namely where the scaling between speed of elongation and the noise strength is such that the rupture occurs late enough so that all of the bonds are stretched close to the deterministic rupture distance, but early enough so that the rupture time is much smaller than the exponentially large time that one would get in a time-independent situation. The importance of that regime is apparent by the fact that in most of the above cited papers (in particular in []) the parameters are chosen such that rupture occurs after a relatively long, but not extremely long time.

The passage from Gaussian to non-Gaussian setting is far from trivial: neither the Gaussian large deviations estimates used in [3,4] nor the symmetry arguments from [1] are available 
any more. The main tool that allows us to succeed is an a priori estimate for the path distance of two stochastic processes, proposition 4, that may be of some interest in its own right. With its help, we can expand the inter-particle potential to second order around the rupture distance and estimate the distance between the original process and its Gaussian approximation by a functional of a Gaussian process. As a result, we recover the results of $[3,4]$ in the nonlinear setting.

Our model fits into the broader picture of systems of interacting Brownian particles. When such systems evolve in a smooth way on large scales, they have been studied before via hydrodynamic limits for many years. For systems at equilibrium, important results include the seminal work [20] on Gaussian fluctuations around reversible equilibria, and the recent significant progress [7] on convergence to the KPZ equation for weakly asymmetric onedimensional Ginzburg-Landau interface models. For the hydrodynamic limit of general nonequilibrium systems, the classical result [21] establishes the hydrodynamic limit for onedimensional systems with repulsive interactions on the torus. In the case of Ginzburg-Landau models, significant progress (in particular, extension to higher dimensions) was achieved in [11].

The situation of a chain rupture is however very different because it is discontinuous on the macroscopic scale. It is the easiest case of the even more challenging problem of crack propagation through solids; these cracks move on a different time scale than the standard macroscopic dynamics, and no rigorous mathematical tools seem to exist as yet for studying them. Numerical studies include $[5,18]$. But even for the one-dimensional case, i.e. the rupture of a molecular chain under strain, the majority of the activity is non-rigorous, including all of the works cited in the first two paragraphs. The only other rigorous approach to chain rupture that we are aware of is the programme by Malyshev and Muzychka [15, 16], where only the mean exit time of the chain is considered, but in the much more difficult situation where the chain is not overdamped.

In the context of the programme started in [1], the work [2] constitutes a first step away from the restrictions of both overdamped dynamics and strictly convex potential. However, the results are again rather limited, as they only hold when the damping diverges as the noise strength goes to zero, and only for a chain of three particles. In particular in view of the results of [], it would be quite interesting to at least remove the restriction on the number of particles.

The paper is structured as follows: in section 2, we state the main result of the paper. An outline of the proof with the most important steps and subresults is given in section 3 . The remaining sections are devoted to the details of the proofs.

\section{Model and main result}

Our model is mathematically equivalent to the one-dimensional Ginzburg-Landau model with a time-dependent boundary condition. Let $d \geqslant 2$ be an integer, and consider a chain of $d+1$ particles located on the real line, interacting via a nearest neighbour ${ }^{3}$ force given by the derivative of a potential $U$. The positions of the particles at time $t$ are denoted by $\mathcal{X}_{t}^{0}, \ldots, \mathcal{X}_{t}^{d}$. We assume that $\mathcal{X}_{0}^{i}=i, \mathcal{X}_{t}^{0}=0$ for all $t$, and $\mathcal{X}_{t}^{d}=\mathcal{X}_{0}^{d}+\varepsilon t$ for $\varepsilon>0$ and all $t$. This means that the leftmost particle is fixed and the rightmost one is pulled with speed $\varepsilon$ to the right. Altogether,

\footnotetext{
${ }^{3}$ We always use the word 'neighbour' to mean consecutive indices, not neighbours in space. This is justified e.g. if we think of the chain as consisting of a string of molecules. On the other hand, in the applications we have in mind particles swap places with negligible probability, in which case there is no difference between spatial and index neighbours.
} 
the model is thus described by the system of stochastic differential equations

$$
\begin{cases}\mathcal{X}_{0}^{i}=i & i=0,1, \ldots, d \\ \mathcal{X}_{t}^{0}=0 & t \geqslant 0 ; \\ \mathcal{X}_{t}^{d}=d+\varepsilon t & t \geqslant 0 ; \\ \mathrm{d} \mathcal{X}_{t}^{i}=\left(U^{\prime}\left(\mathcal{X}_{t}^{i+1}-\mathcal{X}_{t}^{i}\right)-U^{\prime}\left(\mathcal{X}_{t}^{i}-\mathcal{X}_{t}^{i-1}\right)\right) \mathrm{d} t+\sigma \mathrm{d} B_{t}^{i}, & i=1, \ldots, d-1, t \geqslant 0,\end{cases}
$$

where $\left(B_{t}^{i}\right)_{t \geqslant 0}$ are independent Brownian motions, $i=1, \ldots, d-1, \sigma \geqslant 0, \varepsilon \geqslant 0$, and $U$ is a sufficiently regular function. We will often write $\mathcal{X}_{t}:=\left(\mathcal{X}_{t}^{0}, \ldots, \mathcal{X}_{t}^{d}\right)^{\top}$.

We will be interested the asymptotic behaviour of the model as $\varepsilon$ and $\sigma$ vanish. Quantities of interest are the time and location (along the chain) of the chain rupture under the dynamics. The physically most desirable choice for $U$ is certainly a potential of Morse type, attractive at short distances but becoming flat at infinity. This then leads to a motion where at first the chain becomes more and more elongated, until at some point a fluctuation makes one of the gaps between two neighbouring particles so large that it energetically favourable for the chain to split into two disconnected pieces. It is not hard to see that e.g. for potentials $U$ that are strictly convex on an interval containing the starting distance of 1 between two particles, this critical gap size corresponds to the first inflection point of $U$ on $[1, \infty)$, i.e. the smallest value $r$ for which $U^{\prime \prime}(r)=0$.

The investigation of a break at an inflection point poses some difficulties. The reason is that in the intermediate regime that we are interested in, the chain remains in a position where all particles are very nearly evenly spaced right up to the time when it breaks. In such a situation of almost equal distances $s$, a Taylor expansion of $U^{\prime}$ around $s$ shows that the effective force which prevents each particle from leaving the position in the middle of its neighbours is equal to $U^{\prime \prime}(s)$. A first problem is that this vanishes when $s$ approaches the inflection point $r$, and higher order terms of the expansion take over and have to be dealt with. A second problem is that when some fluctuation eventually causes the distance between two particles to exceed the critical distance $r$, another fluctuation may well bring them back closer together before the deterministic part of the dynamics has had enough time to pull the chain apart. It is therefore not even completely clear what the correct definition for a break time should be.

We avoid both problems discussed above by making two crucial assumptions. The first is that there is a fixed distance $\mathfrak{b}>1$ such that the chain breaks whenever two neighbouring particles are $\mathfrak{b}$ or more apart from each other. We thus introduce the stopping times

$$
\tau_{\mathcal{X}, \mathfrak{b}}^{i}=\tau_{\mathcal{X}, \mathfrak{b}}^{i}(\varepsilon, \sigma):=\inf \left\{t \geqslant 0 \mid \mathcal{X}_{t}^{i}-\mathcal{X}_{t}^{i-1}=\mathfrak{b}\right\}
$$

for $i \in\{1, \ldots, d\}$, and

$$
\tau_{\mathcal{X}, \mathfrak{b}}=\tau_{\mathcal{X}, \mathfrak{b}}(\varepsilon, \sigma):=\min _{1 \leqslant q i \leqslant d} \tau_{\mathcal{X}, \mathfrak{b}}^{i}=\inf \left\{t \geqslant 0: \exists i \in\{1, \ldots, d\}: \mathcal{X}_{t}^{i}-\mathcal{X}_{t}^{i-1}=\mathfrak{b}\right\},
$$

and investigate their distributions. Furthermore, we assume that $U$ is strictly convex and increasing up to $\mathfrak{b}$, thus guaranteeing that a particle configuration with equal distances between particles is a stable equilibrium of the no-noise dynamics. From the geometric point of view based on the observation of the process $\left(\mathcal{X}_{t}\right)$, the break time simply means the exit time of $\mathcal{X}$ from a certain deterministic polytope. Accordingly, we call $\tau_{\mathcal{X}, \mathfrak{b}}^{i}, \tau_{\mathcal{X}, \mathfrak{b}}$, and other similar variables exit times. 
The convexity assumption on $U$ is made explicit via

Assumption P. The function $U$ is three times continuously differentiable and $U^{\prime \prime}$ is strictly positive on $[1, \mathfrak{b}]$.

The scaling regime we are interested in is characterized by the conditions

$$
\sigma / \varepsilon \rightarrow \infty \quad \text { and } \quad \sigma^{2}|\ln \varepsilon|^{3} \rightarrow 0
$$

on the scaling parameters $\sigma$ and $\varepsilon$. This is very slightly smaller than the 'intermediate regime' found in [3, 4], the difference being owed to the necessity to compare a Gaussian to the relevant non-Gaussian process. For stating our main result, we define the quantities

$$
\begin{aligned}
& v^{2}:=\frac{d-1}{2 d}, \quad \gamma:=\sqrt{2} d v=\sqrt{d(d-1)}, \\
& A_{1}:=A_{d}:=\frac{d}{d-1}, \quad A_{i}:=\frac{2 d}{d-1}, \quad i \in\{2, \ldots, d-1\}, \\
& a_{i}:=v d A_{i} / \sqrt{2 \pi} \quad \text { for } i \in\{1, \ldots, d\}, \\
& a_{0}:=\sum_{i=1}^{d} a_{i}=2 v d^{2} / \sqrt{2 \pi}, \quad b:=\sqrt{2} /(v d) .
\end{aligned}
$$

Recall that a random variable $\chi$ is double exponential (or Gumbel) with parameters $a, b>0$, if

$$
\mathbb{P}(\chi \leqslant r)=\exp (-a \exp (-b r)), \quad r \in \mathbb{R} .
$$

Below and in the remainder of the paper, we write $\Rightarrow$ for convergence in distribution.

Theorem 1. Let $\left(\mathcal{X}_{t}^{i}\right)_{i=0, \ldots, d}$ solve the system (1), where the potential $U$ satisfies assumption P. Let $\mathfrak{b}>1$, set $\mathfrak{u}:=U^{\prime \prime}(\mathfrak{b})$, and define $\tau_{\mathcal{X}, \mathfrak{b}}^{i}, \tau_{\mathcal{X}, \mathfrak{b}}$ and $t^{*}(\varepsilon)$ as in (2), (3) and (9), respectively. Then in the parameter regime described by (4), we have the following weak limit theorems for the break times as $\varepsilon, \sigma \rightarrow 0$ :

$$
\begin{array}{r}
\frac{\sqrt{\mathfrak{u}} \varepsilon}{\sigma} \sqrt{\ln (\sigma / \varepsilon)}\left(t_{*}(\varepsilon, \mathfrak{b})-\gamma \frac{\sigma}{\sqrt{\mathfrak{u}} \varepsilon} \sqrt{\ln (\sigma / \varepsilon)}-\tau_{\mathcal{X}, \mathfrak{b}}^{i}(\varepsilon, \sigma)\right) \Rightarrow \chi_{i}(\mathfrak{u}), \\
i \in\{1, \ldots, d\},
\end{array}
$$

and

$$
\frac{\sqrt{\mathfrak{u}} \varepsilon}{\sigma} \sqrt{\ln (\sigma / \varepsilon)}\left(t_{*}(\varepsilon, \mathfrak{b})-\gamma \frac{\sigma}{\sqrt{\mathfrak{u}} \varepsilon} \sqrt{\ln (\sigma / \varepsilon)}-\tau_{\mathcal{X}, \mathfrak{b}}(\varepsilon, \sigma)\right) \Rightarrow \chi_{0}(\mathfrak{u}),
$$

where for each $i, \chi_{i}(\mathfrak{u})$ is a double exponential random variable with parameters $\sqrt{\mathfrak{u}} a_{i}, b$, respectively.

Moreover, under the same assumptions, we have

$$
\mathbb{P}\left(\tau_{\mathcal{X}, \mathfrak{b}}=\tau_{\mathcal{X}, \mathfrak{b}}^{i}\right) \rightarrow \begin{cases}\frac{1}{d-1} & i \in\{2, \ldots, d-1\} \\ \frac{1}{2(d-1)} & i \in\{1, d\}\end{cases}
$$

as $\sigma, \varepsilon \rightarrow 0$. 
Theorem 1 corresponds to the result in the intermediate regime found in [3, 4]. It contains an interesting universality in the sense that the asymptotic break time distribution only depends on the curvature $U^{\prime \prime}(\mathfrak{b})$ of $U$ at the breaking distance. The reason for this universality is not hard to understand intuitively. Since the chain (of initial length $d$ ) must break once its total length exceeds $d \mathfrak{b}$, there is the simple, but important bound

$$
\tau_{\mathcal{X}, \mathfrak{b}} \leqslant t_{*}=t_{*}(\varepsilon, \mathfrak{b}):=d(\mathfrak{b}-1) / \varepsilon
$$

For the intermediate pulling regime, the break actually occurs when the chain is quite close to the maximal allowed elongation $d \mathfrak{b}$, and is therefore initiated by rather small fluctuations around the stable equilibrium. Therefore, the quadratic approximation to the potential is a good one for this situation, and we obtain both the universality and the actual result by comparison with the relevant Gaussian processes. That said, the rigorous implementation of such comparisons on the level of sample paths is technically not easy. We achieve it by the a priori estimate proposition 4 , which should be considered the main technical contribution of the present paper.

\section{Outline of the proof}

The first step in the proof of theorem 1 consists in a slight generalization of the relevant result in [3]. Put very succinctly, it states that the statement of theorem 1 holds in the special case $U(x)=\mathfrak{u} x^{2} / 2$ with $\mathfrak{u}>0$. Since we will need the notation in the proof later on anyway, we spell out the statement here.

Consider the linear system

$$
\begin{cases}X_{0}^{i}=i & i=0,1, \ldots, d ; \\ X_{t}^{0}=0 & t \geqslant 0 ; \\ X_{t}^{d}=d+\varepsilon t & t \geqslant 0 ; \\ \mathrm{d} X_{t}^{i}=\mathfrak{u}\left(X_{t}^{i+1}+X_{t}^{i-1}-2 X_{t}^{i}\right) \mathrm{d} t+\sigma \mathrm{d} B_{t}^{i}, & i=1, \ldots, d-1, t \geqslant 0,\end{cases}
$$

where $\mathfrak{u}>0$ is a constant and we will abbreviate $\boldsymbol{X}_{t}:=\left(X_{t}^{0}, \ldots, X_{t}^{d}\right)^{\top}$. As before, define the break times as

$$
\tau^{i}=\tau^{i}(\varepsilon, \sigma):=\inf \left\{t \geqslant 0 \mid X_{t}^{i}-X_{t}^{i-1}=\mathfrak{b}\right\}
$$

and

$$
\tau=\tau(\varepsilon, \sigma):=\min _{1 \leqslant i \leqslant d} \tau^{i}=\inf \left\{t \geqslant 0: \exists i \in\{1, \ldots, d\}: X_{t}^{i}-X_{t}^{i-1}=\mathfrak{b}\right\}
$$

When we want to stress the dependence on the parameters $\mathfrak{u}$ and $\mathfrak{b}$, we will write $\boldsymbol{X}_{\mathfrak{u}, t}, X_{\mathfrak{u}, t}^{i}$, $\tau_{\mathfrak{u}, \mathfrak{b}}^{i}(\varepsilon, \sigma)$ and $\tau_{\mathfrak{u}, \mathfrak{b}}(\varepsilon, \sigma)$.

We consider scaling regime where

$$
\sigma / \varepsilon \rightarrow \infty \quad \text { and } \quad \sigma^{2}|\ln \varepsilon| \rightarrow 0
$$

Note that this regime is slightly wider than the one given in (4); the small difference is due to 
the fact that close to the large deviation regime, the Gaussian and the non-Gaussian processes start to look differently. In this regime, we have

Theorem 2. Assume that (11) holds. Then, as $\varepsilon, \sigma \rightarrow 0$, the analogue of (8) holds for $\tau_{\mathfrak{u}, \mathfrak{b}}(\varepsilon, \sigma)$ and $\tau_{\mathfrak{u}, \mathfrak{b}}^{i}(\varepsilon, \sigma)$ and we have the following weak limit theorems for the break times:

$\frac{\sqrt{\mathfrak{u}} \varepsilon}{\sigma} \sqrt{\ln (\sigma / \varepsilon)}\left(t_{*}(\varepsilon, \mathfrak{b})-\gamma \frac{\sigma}{\sqrt{\mathfrak{u}} \varepsilon} \sqrt{\ln (\sigma / \varepsilon)}-\tau_{\mathfrak{u}, \mathfrak{b}}^{i}(\varepsilon, \sigma)\right) \Rightarrow \chi_{i}(\mathfrak{u}), i \in\{1, \ldots, d\}$,

and

$$
\frac{\sqrt{\mathfrak{u}} \varepsilon}{\sigma} \sqrt{\ln (\sigma / \varepsilon)}\left(t_{*}(\varepsilon, \mathfrak{b})-\gamma \frac{\sigma}{\sqrt{\mathfrak{u}} \varepsilon} \sqrt{\ln (\sigma / \varepsilon)}-\tau_{\mathfrak{u}, \mathfrak{b}}(\varepsilon, \sigma)\right) \Rightarrow \chi_{0}(\mathfrak{u}),
$$

where $\chi_{i}(\mathfrak{u})$ is a double exponential random variable with parameters $\sqrt{\mathfrak{u}} a_{i}, b$, and the values $a_{i}, b$ are defined in (5).

[3] contains theorem 2 for the special case $\mathfrak{b}=2, \mathfrak{u}=1$. The passage to general $\mathfrak{b}$ and $\mathfrak{u}$ is made by standard scaling arguments, which we spell out in section 4 for the convenience of the reader.

The second step is to replace $\mathfrak{u}$ in the system (10) by a time-dependent quantity $\phi(t)$. Again, the short version of the result is that the statement of theorem 2 remains true with $\mathfrak{u}$ replaced by $\phi(t)$, but we spell out the result as we will need the notation later in the proofs anyway.

Let $\boldsymbol{Z}_{t}=\left(Z_{t}^{0}, \ldots, Z_{t}^{d}\right)^{\top}$ solve the linear system with time-dependent coefficient

$$
\begin{cases}Z_{0}^{i}=i & i=0,1, \ldots, d \\ Z_{t}^{0}=0 & t \geqslant 0 \\ Z_{t}^{d}=d+\varepsilon t & t \geqslant 0 \\ \mathrm{~d} Z_{t}^{i}=\phi(t)\left(Z_{t}^{i+1}+Z_{t}^{i-1}-2 Z_{t}^{i}\right) \mathrm{d} t+\sigma \mathrm{d} B_{t}^{i}, & i=1, \ldots, d-1, t \geqslant 0\end{cases}
$$

Here we immediately take $\phi(t):=U^{\prime \prime}\left(q_{t}\right)$ with

$$
q_{t}:=1+\varepsilon \frac{t}{d}
$$

where $U$ fulfils assumption P. This is the correct linearization of (1) in the following sense: by the assumptions on $U$, the potential energy $\boldsymbol{U}(x)=\sum_{i=1}^{d} U\left(x_{i}-x_{i-1}\right)$ is minimized by the vector $\left(i\left(1+\varepsilon \frac{t}{d}\right)\right)_{0 \leqslant i \leqslant d}$ at time $t$. Since the pulling is slow and the noise is small, the system will be close to that energy minimum at all times, and as terms of order zero and one cancel when we Taylor expand each $U\left(x_{i}-x_{i-1}\right)$ around $1+\varepsilon \frac{t}{d}$, so that $\phi(t)=U^{\prime \prime}\left(1+\varepsilon \frac{t}{d}\right)$ is the dominant term. Notice in particular that for quadratic potentials $U(x)=\mathfrak{u} x^{2} / 2$, we have $\phi(\cdot) \equiv \mathfrak{u}$ and $\left(\boldsymbol{Z}_{t}\right)$ coincides with $\left(\boldsymbol{X}_{\mathfrak{u}, t}\right)$.

Since $\boldsymbol{Z}=\left(Z^{0}, \ldots, Z^{d}\right)^{\top}$ is still a Gaussian process, we will be able to analyse it in great detail. We work in the scaling regime

$$
\sigma / \varepsilon \rightarrow \infty \text { and } \sigma^{2}|\ln \varepsilon|^{3 / 2} \rightarrow 0
$$

which is larger than the one for theorem 1 but smaller than the one for theorem 2 due to our need to accommodate the fact that the coefficient of the linear force now depends on time. As above, we introduce the break times

$$
\tau_{Z, \mathfrak{b}}^{i}=\tau_{Z, \mathfrak{b}}^{i}(\varepsilon, \sigma):=\inf \left\{t \geqslant 0 \mid Z_{t}^{i}-Z_{t}^{i-1}=\mathfrak{b}\right\}
$$


and

$$
\tau_{Z, \mathfrak{b}}=\tau_{Z, \mathfrak{b}}(\varepsilon, \sigma):=\min _{1 \leqslant i \leqslant d} \tau_{Z, \mathfrak{b}}^{i}=\inf \left\{t \geqslant 0: \exists i \in\{1, \ldots, d\}: Z_{t}^{i}-Z_{t}^{i-1}=\mathfrak{b}\right\}
$$

We have

Theorem 3. Assume that (15) holds. Fix $\mathfrak{b}>1$ and set $\mathfrak{u}:=U^{\prime \prime}(\mathfrak{b})$. Then, as $\varepsilon, \sigma \rightarrow 0$, the analogue of $(8)$ holds for $\tau_{Z, \mathfrak{b}}(\varepsilon, \sigma)$ and $\tau_{Z, \mathfrak{b}}^{i}(\varepsilon, \sigma)$ and we have the following weak limit theorems for the break times:

$\frac{\sqrt{\mathfrak{u}} \varepsilon}{\sigma} \sqrt{\ln (\sigma / \varepsilon)}\left(t_{*}(\varepsilon, \mathfrak{b})-\gamma \frac{\sigma}{\sqrt{\mathfrak{u}} \varepsilon} \sqrt{\ln (\sigma / \varepsilon)}-\tau_{Z, \mathfrak{b}}^{i}(\varepsilon, \sigma)\right) \Rightarrow \chi_{i}(\mathfrak{u}), i \in\{1, \ldots, d\}$,

and

$$
\frac{\sqrt{\mathfrak{u}} \varepsilon}{\sigma} \sqrt{\ln (\sigma / \varepsilon)}\left(t_{*}(\varepsilon, \mathfrak{b})-\gamma \frac{\sigma}{\sqrt{\mathfrak{u}} \varepsilon} \sqrt{\ln (\sigma / \varepsilon)}-\tau_{Z, \mathfrak{b}}(\varepsilon, \sigma)\right) \Rightarrow \chi_{0}(\mathfrak{u}),
$$

where $\chi_{i}(\mathfrak{u})$ is a double exponential random variable with parameters $\sqrt{\mathfrak{u}} a_{i}, b$, and $a_{i}, b$ are defined in (5).

The third step is an a priori estimate that allows to estimate the path distance between the processes $\mathcal{X}$ and $\boldsymbol{Z}$ on the relevant time scales. We assume that $\boldsymbol{Z}$ and $\mathcal{X}$ are defined on the same probability space, and are driven by the same Brownian motions. On this probability space we define the stochastic processes

$$
S_{t}^{*}:=\sup _{0 \leqslant s \leqslant t}\left\|\boldsymbol{Z}_{s}-\mathcal{X}_{s}\right\|_{2}=\sup _{0 \leqslant s \leqslant t}\left(\sum_{i=0}^{d}\left|Z_{s}^{i}-\mathcal{X}_{s}^{i}\right|^{2}\right)^{1 / 2}=\sup _{0 \leqslant s \leqslant t}\left(\sum_{i=1}^{d-1}\left|Z_{s}^{i}-\mathcal{X}_{s}^{i}\right|^{2}\right)^{1 / 2}
$$

and

$$
M_{t}^{*}:=\sup _{0 \leqslant s \leqslant t}\left(\sum_{i=1}^{d}\left|Z_{s}^{i}-Z_{s}^{i-1}-q_{s}\right|^{2}\right)^{1 / 2},
$$

where we recall that $q_{s}=1+\frac{\varepsilon s}{d}$. We then have

Proposition 4. Let $r$ be as in assumption $\mathrm{P}$. Then there exists a large constant $C$ depending on $d$ and on the potential $U$ such that for all $t \leqslant t_{*}$ and all $\delta>0$ we have

$$
\mathbb{P}\left(S_{t}^{*} \geqslant \delta\right) \leqslant \mathbb{P}\left(M_{t}^{*} \geqslant \sqrt{\delta} / C\right)+\mathbb{P}\left(M_{t}^{*}+4 C\left(M_{t}^{*}\right)^{2} \geqslant r\right)+\mathbb{P}\left(C M_{t}^{*} \geqslant 1\right) .
$$

The details of the proof of theorem 1 are given in the following sections and are organized as follows. Theorem 2 is proved in section 4, while theorem 3 is proved in section 5 . It is used, together with proposition 4 , to prove theorem 1 in section 6 . The proof of proposition 4 and other more technical proofs are collected in section 7 .

\section{Scaling of the standard model}

Here we prove theorem 2. Recall that the case where $\mathfrak{u}=1, \mathfrak{b}=2$ has been done in [3]. Below, we will refer to this case as the standard problem. 
Step 1. Scaling of $\mathfrak{u}$. We first consider the scaling of $\mathfrak{u}$ with fixed $\mathfrak{b}$. Let $\widetilde{\boldsymbol{X}}$ be a solution of the system (10) with parameters $\mathfrak{u}=1, \widetilde{\varepsilon}$ and $\widetilde{\sigma}$. We make the time change

$$
\widehat{X}_{t}^{i}:=\widetilde{X}_{\mathfrak{u} t}^{i}
$$

This family solves the system with parameters $\mathfrak{u}, \varepsilon:=\mathfrak{u} \widetilde{\varepsilon}$, and $\sigma:=\sqrt{\mathfrak{u}} \widetilde{\sigma}$, because $\widehat{X}_{t}^{0}=\widetilde{X}_{\mathfrak{u} t}^{0}=$ $0, \widehat{X}_{t}^{d}=\widetilde{X}_{\mathfrak{u} t}^{d}=d+\widetilde{\varepsilon}(\mathfrak{u} t)=d+(\mathfrak{u} \widetilde{\varepsilon}) t$, and an easy calculation shows that

$$
\mathrm{d} \widehat{X}_{t}^{i}=\mathfrak{u}\left(\widehat{X}_{t}^{i+1}+\widehat{X}_{t}^{i-1}-2 \widehat{X}_{t}^{i}\right) \mathrm{d} t+\sqrt{\mathfrak{u}} \widetilde{\sigma} \mathrm{d} \widehat{B}_{t}^{i}, \quad i=1, \ldots, d-1, t \geqslant 0,
$$

with appropriate independent Brownian motions $\widehat{B}_{t}^{i}$.

The above argument shows that for given $\varepsilon, \sigma$, we have

$$
\boldsymbol{X}_{\mathfrak{u}, t}(\varepsilon, \sigma) \stackrel{d}{=} \boldsymbol{X}_{1, \mathfrak{u} t}\left(\frac{\varepsilon}{\mathfrak{u}}, \frac{\sigma}{\sqrt{\mathfrak{u}}}\right)
$$

the latter being a solution of the standard problem, yet with modified parameters.

Next, having a relation between the processes, we obtain the relations between the break times for arbitrary $\mathfrak{b}>1$,

$$
\begin{gathered}
\tau_{\mathfrak{u}, \mathfrak{b}}^{i}(\varepsilon, \sigma)=\mathfrak{u}^{-1} \tau_{1, \mathfrak{b}}^{i}(\varepsilon / \mathfrak{u}, \sigma / \sqrt{\mathfrak{u}}), \\
\tau_{\mathfrak{u}, \mathfrak{b}}(\varepsilon, \sigma)=\mathfrak{u}^{-1} \tau_{1, \mathfrak{b}}(\varepsilon / \mathfrak{u}, \sigma / \sqrt{\mathfrak{u}}) .
\end{gathered}
$$

Step 2. Scaling of $\mathfrak{b}$. Now we fix $\mathfrak{u}=1$ and scale $\mathfrak{b}$. Let us fix a break position $i \in\{1, \ldots, d\}$. The break condition is

$$
X_{t}^{i}-X_{t}^{i-1}=\mathfrak{b}
$$

This is equivalent to

$$
\sigma V_{t}^{i}+\varepsilon \frac{t}{d}+\varepsilon R_{t}^{i}=\mathfrak{b}-1
$$

where $V^{i}$ is an appropriate asymptotically stationary process, and $R^{i}$ is a bounded deterministic function, both defined in [3]. Dividing by $\mathfrak{b}-1$ yields the equivalent form

$$
\frac{\sigma}{\mathfrak{b}-1} V_{t}^{i}+\frac{\varepsilon}{\mathfrak{b}-1} \frac{t}{d}+\frac{\varepsilon}{\mathfrak{b}-1} R_{t}^{i}=1
$$

which coincides with the break condition for the standard case $\mathfrak{b}=2$ with parameters $\frac{\varepsilon}{1-\mathfrak{b}}, \frac{\sigma}{1-\mathfrak{b}}$. We conclude that $\tau_{1, \mathfrak{b}}^{i}(\varepsilon, \sigma)$ coincides in distribution with $\tau_{1,2}^{i}\left(\frac{\varepsilon}{\mathfrak{b}-1}, \frac{\sigma}{\mathfrak{b}-1}\right)$.

Step 3. Combining two scalings. By combining the results of two scalings we see that the vector $\tau_{\mathfrak{u}, \mathfrak{b}}^{i}(\varepsilon, \sigma), i=1, \ldots, d$, has the same distribution as the vector $\left.\mathfrak{u}^{-1} \tau_{1,2}^{i}\left(\frac{\varepsilon}{\mathfrak{u}(\mathfrak{b}-1)}, \frac{\sigma}{\sqrt{\mathfrak{u}(\mathfrak{b}-1)}}\right)\right), i=1, \ldots, d$. Subsequently, this also holds for $\tau_{\mathfrak{u}, \mathfrak{b}}=\min _{i} \tau_{\mathfrak{u}, \mathfrak{b}}^{i}$ and $\tau_{1,2}=\min _{i} \tau_{1,2}^{i}$.

Step 4. Weak convergence. From [3] we know that theorem 2 is valid in the standard problem. We apply this version of the theorem to the modified pair of parameters $\left.\left(\frac{\varepsilon}{\mathfrak{u}(\mathfrak{b}-1)}, \frac{\sigma}{\sqrt{\mathfrak{u}(b-1)}}\right)\right)$ and obtain

$$
\frac{\varepsilon}{\sigma \sqrt{\mathfrak{u}}} \sqrt{\ln \left(\frac{\sigma \sqrt{\mathfrak{u}}}{\varepsilon}\right)}\left(\frac{d \mathfrak{u}(\mathfrak{b}-1)}{\varepsilon}-\gamma \frac{\sigma \sqrt{\mathfrak{u}}}{\varepsilon} \sqrt{\ln \left(\frac{\sigma \sqrt{\mathfrak{u}}}{\varepsilon}\right)}-\mathfrak{u} \tau_{\mathfrak{u}, \mathfrak{b}}^{i}(\varepsilon, \sigma)\right) \Rightarrow \chi_{i},
$$


or, equivalently,

$$
\frac{\varepsilon \sqrt{\mathfrak{u}}}{\sigma} \sqrt{\ln \left(\frac{\sigma \sqrt{\mathfrak{u}}}{\varepsilon}\right)}\left(\frac{d(\mathfrak{b}-1)}{\varepsilon}-\gamma \frac{\sigma}{\varepsilon \sqrt{\mathfrak{u}}} \sqrt{\ln \left(\frac{\sigma \sqrt{\mathfrak{u}}}{\varepsilon}\right)}-\tau_{\mathfrak{u}, \mathfrak{b}}^{i}(\varepsilon, \sigma)\right) \Rightarrow \chi_{i} .
$$

Next, using expansion

$$
\begin{aligned}
\sqrt{\ln \left(\frac{\sigma \sqrt{\mathfrak{u}}}{\varepsilon}\right)} & =\sqrt{\ln \left(\frac{\sigma}{\varepsilon}\right)+\frac{\ln \mathfrak{u}}{2}}=\sqrt{\ln \left(\frac{\sigma}{\varepsilon}\right)} \sqrt{1+\frac{\ln \mathfrak{u}}{2 \ln \left(\frac{\sigma}{\varepsilon}\right)}} \\
& =\sqrt{\ln \left(\frac{\sigma}{\varepsilon}\right)}\left(1+\frac{\ln \mathfrak{u}}{4 \ln \left(\frac{\sigma}{\varepsilon}\right)}(1+o(1))\right) \\
& =\sqrt{\ln \left(\frac{\sigma}{\varepsilon}\right)}+\frac{\ln \mathfrak{u}}{4 \sqrt{\ln \left(\frac{\sigma}{\varepsilon}\right)}}(1+o(1))
\end{aligned}
$$

we obtain

$$
\frac{\varepsilon \sqrt{\mathfrak{u}}}{\sigma} \sqrt{\ln \left(\frac{\sigma}{\varepsilon}\right)}\left(\frac{d(\mathfrak{b}-1)}{\varepsilon}-\gamma \frac{\sigma}{\varepsilon \sqrt{\mathfrak{u}}} \sqrt{\ln \left(\frac{\sigma}{\varepsilon}\right)}-\tau_{\mathfrak{u}, \mathfrak{b}}^{i}(\varepsilon, \sigma)\right) \Rightarrow \chi_{i}+\frac{\gamma \ln \mathfrak{u}}{4} .
$$

Notice that a shifted variable having a double exponential distribution belongs to the same class: if $\kappa \in \mathbb{R}$ and $\xi$ is double exponential with parameters $a, b$, then $\xi+\kappa$ is also double exponential with parameters $a \exp \{b \kappa\}, b$.

In our case, $\kappa=\frac{\gamma \ln \mathfrak{u}}{4}$, hence $\exp \{b \kappa\}=\mathfrak{u}^{b \gamma / 4}$. Furthermore, an easy calculation shows that $b \gamma=\sqrt{2}(v d)^{-1} \cdot \sqrt{2} \mathrm{~d} v=2$. Therefore, $\exp \{b \kappa\}=\sqrt{\mathfrak{u}}$. We finally obtain

$$
\frac{\varepsilon \sqrt{\mathfrak{u}}}{\sigma} \sqrt{\ln \left(\frac{\sigma}{\varepsilon}\right)}\left(\frac{d(\mathfrak{b}-1)}{\varepsilon}-\gamma \frac{\sigma}{\varepsilon \sqrt{\mathfrak{u}}} \sqrt{\ln \left(\frac{\sigma}{\varepsilon}\right)}-\tau_{\mathfrak{u}, \mathfrak{b}}^{i}(\varepsilon, \sigma)\right) \Rightarrow \chi_{i}(\mathfrak{u}),
$$

where $\chi_{i}(\mathfrak{u})$ is double exponential with parameters $\sqrt{\mathfrak{u}} a_{i}, b$.

The latter equation coincides with the claim (12). The proof of (13) is exactly the same. Relation (8) follows as a by-product of the scaling reductions. Therefore, the proof of theorem 2 is finished.

Remark 5. We see from the proof that the weak convergence in (12) and (13) is locally uniform in $\mathfrak{b}$ (as long as $\mathfrak{b}$ is bounded away from 1 ) for every fixed $\mathfrak{u}$. We will use this fact later on.

\section{Comparing the Gaussian processes $X_{\mathfrak{u}, \mathfrak{b}}$ and $Z$}

Before we start with the proof of theorem 3, we need two auxiliary results. The first one shows that $\boldsymbol{Z}$ has no early breaks. Recall the notation $t_{*}=t_{*}(\varepsilon, \mathfrak{b}):=d(\mathfrak{b}-1) / \varepsilon$.

Lemma 6. There exists $\gamma_{1}=\gamma_{1}(d, U(\cdot))$ such that

$$
\mathbb{P}\left(\tau_{Z, \mathfrak{b}}(\varepsilon, \sigma)<t_{*}-\gamma_{1} \frac{\sigma}{\varepsilon} \sqrt{\ln \left(\frac{\sigma}{\varepsilon}\right)}\right) \rightarrow 0, \quad \text { as } \varepsilon, \quad \sigma \rightarrow 0 .
$$

From theorem 2 we know that the same statement is true for $\boldsymbol{X}_{\mathfrak{u}}$, namely, for any $\gamma_{1}>\gamma / \sqrt{\mathfrak{u}}$, 


$$
\mathbb{P}\left(\tau_{\mathfrak{u}, \mathfrak{b}}(\varepsilon, \sigma)<t_{*}-\gamma_{1} \frac{\sigma}{\varepsilon} \sqrt{\ln \left(\frac{\sigma}{\varepsilon}\right)}\right) \rightarrow 0
$$

But we also need (22) for $\boldsymbol{Z}$. The proof of lemma 6 is given in section 7.2.

The second auxiliary lemma shows that $\boldsymbol{Z}$ and $\boldsymbol{X}_{\mathfrak{u}}$ are close on an important time interval.

Lemma 7. Let $\gamma_{1}>0$ and $\theta>0$. Assume (15). Denote $\delta=\delta(\varepsilon, \sigma):=\frac{\theta \sigma}{\sqrt{\ln \left(\frac{\sigma}{\varepsilon}\right)}}$. Then, as $\varepsilon, \sigma \rightarrow 0$, for every $i=0,1, \ldots, d$, we have

$$
\mathbb{P}\left(\sup _{t \in\left[t_{*}-\gamma_{1} \frac{\sigma}{\varepsilon} \sqrt{\ln \left(\frac{\sigma}{\varepsilon}\right)}, t_{*}\right]}\left|Z_{t}^{i}-X_{\mathfrak{u}, t}^{i}\right|>\delta\right) \rightarrow 0 .
$$

The proof of lemma 7 is given in section 7.3.

Proof of theorem 3. Let $\gamma_{1}$ be chosen large enough to satisfy lemma 6 . Let $t \in\left[t_{*}-\right.$ $\left.\gamma_{1} \frac{\sigma}{\varepsilon} \sqrt{\ln \left(\frac{\sigma}{\varepsilon}\right)}, t_{*}\right]$ be such that

$$
\max _{1 \leqslant i \leqslant d}\left(Z_{t}^{i}-Z_{t}^{i-1}\right) \geqslant \mathfrak{b}
$$

If, additionally,

$$
\max _{0 \leqslant i \leqslant d}\left|Z_{t}^{i}-X_{\mathfrak{u}, t}^{i}\right|<\delta
$$

then

$$
\max _{1 \leqslant i \leqslant d}\left(X_{\mathfrak{u}, t}^{i}-X_{\mathfrak{u}, t}^{i-1}\right) \geqslant \mathfrak{b}-2 \delta
$$

It follows that $\tau_{\mathfrak{u}, \mathfrak{b}-2 \delta} \leqslant t$. By using lemmas 6 and 7 we obtain

$$
\mathbb{P}\left(\tau_{Z, \mathfrak{b}} \leqslant t\right) \leqslant \mathbb{P}\left(\tau_{\mathfrak{u}, \mathfrak{b}-2 \delta} \leqslant t\right)+o(1)
$$

with $o(1)$ uniformly over $t \in\left[t_{*}-\gamma_{1} \frac{\sigma}{\varepsilon} \sqrt{\ln \left(\frac{\sigma}{\varepsilon}\right)}, t_{*}\right]$.

Let $r \in \mathbb{R}$ be fixed. The previous equation and the definition of $\delta$ yield

$$
\begin{aligned}
P_{r}:= & \mathbb{P}\left(\frac{\sqrt{\mathfrak{u}} \varepsilon}{\sigma} \sqrt{\ln (\sigma / \varepsilon)}\left(t_{*}-\gamma \frac{\sigma}{\sqrt{\mathfrak{u}} \varepsilon} \sqrt{\ln (\sigma / \varepsilon)}-\tau_{Z, \mathfrak{b}}(\varepsilon, \sigma)\right) \geqslant r\right) \\
= & \mathbb{P}\left(\tau_{Z, \mathfrak{b}}(\varepsilon, \sigma) \leqslant t_{*}-\gamma \frac{\sigma}{\sqrt{\mathfrak{u}} \varepsilon} \sqrt{\ln (\sigma / \varepsilon)}-\frac{r \sigma}{\sqrt{\mathfrak{u}} \varepsilon \sqrt{\ln (\sigma / \varepsilon)}}\right) \\
\leqslant & \mathbb{P}\left(\tau_{\mathfrak{u}, \mathfrak{b}-2 \delta}(\varepsilon, \sigma) \leqslant t_{*}-\gamma \frac{\sigma}{\sqrt{\mathfrak{u}} \varepsilon} \sqrt{\ln (\sigma / \varepsilon)}-\frac{r \sigma}{\sqrt{\mathfrak{u}} \varepsilon \sqrt{\ln (\sigma / \varepsilon)}}\right)+o(1) \\
= & \mathbb{P}\left(\tau_{\mathfrak{u}, \mathfrak{b}-2 \delta}(\varepsilon, \sigma) \leqslant \frac{d(\mathfrak{b}-2 \delta-1)}{\varepsilon}+\frac{2 \mathrm{~d} \delta}{\varepsilon}\right. \\
& \left.-\gamma \frac{\sigma}{\sqrt{\mathfrak{u}} \varepsilon} \sqrt{\ln (\sigma / \varepsilon)}-\frac{r \sigma}{\sqrt{\mathfrak{u}} \varepsilon \sqrt{\ln (\sigma / \varepsilon)}}\right)+o(1) . \\
= & \mathbb{P}\left(\tau_{\mathfrak{u}, \mathfrak{b}-2 \delta}(\varepsilon, \sigma) \leqslant t_{*}(\varepsilon, \mathfrak{b}-2 \delta)-\gamma \frac{\sigma}{\sqrt{\mathfrak{u}} \varepsilon} \sqrt{\ln (\sigma / \varepsilon)}-\frac{(r+2 \mathrm{~d} \theta \sqrt{\mathfrak{u}}) \sigma}{\sqrt{\mathfrak{u}} \varepsilon \sqrt{\ln (\sigma / \varepsilon)}}\right)+o(1) .
\end{aligned}
$$




$$
\begin{aligned}
& =\mathbb{P}\left(\frac{\sqrt{\mathfrak{u}} \varepsilon}{\sigma} \sqrt{\ln (\sigma / \varepsilon)}\left(t_{*}(, \varepsilon, \mathfrak{b}-2 \delta)-\gamma \frac{\sigma}{\sqrt{\mathfrak{u}} \varepsilon} \sqrt{\ln (\sigma / \varepsilon)}-\tau_{\mathfrak{u}, \mathfrak{b}-2 \delta}(\varepsilon, \sigma)\right)\right. \\
& \geqslant r+2 \mathrm{~d} \theta \sqrt{\mathfrak{u}})+o(1) .
\end{aligned}
$$

By applying theorem 2 with $\widetilde{\mathfrak{b}}:=\mathfrak{b}-2 \delta$ instead of $\mathfrak{b}$, we obtain ${ }^{4}$

$$
\limsup _{\varepsilon, \sigma \rightarrow 0} P_{r} \leqslant \mathbb{P}\left(\chi_{0}(\mathfrak{u}) \geqslant r+2 \mathrm{~d} \theta \sqrt{\mathfrak{u}}\right) .
$$

Finally, by letting $\theta \rightarrow 0$ we obtain the desired upper bound

$$
\limsup _{\varepsilon, \sigma \rightarrow 0} P_{r} \leqslant \mathbb{P}\left(\chi_{0}(\mathfrak{u}) \geqslant r\right) .
$$

The lower bound follows by the same lines. If for some $t \in\left[t_{*}-\gamma_{1} \frac{\sigma}{\varepsilon} \sqrt{\ln \left(\frac{\sigma}{\varepsilon}\right)}, t_{*}\right]$ we have

$$
\max _{1 \leqslant i \leqslant d}\left(X_{\mathfrak{u}, t}^{i}-X_{\mathfrak{u}, t}^{i-1}\right) \geqslant \mathfrak{b}+2 \delta
$$

and (25) holds, then

$$
\max _{1 \leqslant i \leqslant d}\left(Z_{t}^{i}-Z_{t}^{i-1}\right) \geqslant \mathfrak{b}
$$

In other words, $\tau_{Z, \mathfrak{b}} \leqslant t$.

By using (23) and lemma 7 we obtain

$$
\mathbb{P}\left(\tau_{Z, \mathfrak{b}} \leqslant t\right) \geqslant \mathbb{P}\left(\tau_{\mathfrak{u}, \mathfrak{b}+2 \delta} \leqslant t\right)-o(1),
$$

and the rest of the derivation leading to

$$
\liminf _{\varepsilon, \sigma \rightarrow 0} P_{r} \geqslant \mathbb{P}\left(\chi_{0}(\mathfrak{u}) \geqslant r\right) .
$$

continues as above, $\mathfrak{b}-2 \delta$ being replaced with $\mathfrak{b}+2 \delta$.

\section{Break times for the non-linear system}

We recall the notation used in proposition 4 . The consequence of that proposition that we will need for the proof of our main result is

Proposition 8. Assume that (4) holds. Let $\theta>0$ and $\delta=\delta(\varepsilon, \sigma):=\frac{\theta \sigma}{\sqrt{\ln \left(\frac{\sigma}{\varepsilon}\right)}}$. Then

$$
\lim _{\varepsilon, \sigma \rightarrow 0} \mathbb{P}\left(S_{t_{*}}^{*} \geqslant \delta\right)=0 .
$$

The proof of this proposition is given in section 7.5.

\footnotetext{
${ }^{4}$ We stress that $\widetilde{\mathfrak{b}}$ slightly depends of $\varepsilon, \sigma$ through $\delta$. Therefore, we need a uniform version of the theorem, cf remark 5 .
} 
Proof of theorem 1. Lower bound. Assume that for some $s$ we have

$$
\max _{1 \leqslant i \leqslant d}\left(X_{\mathfrak{u}, s}^{i}-X_{\mathfrak{u}, s}^{i-1}\right) \geqslant \mathfrak{b}+4 \delta, \quad \max _{0 \leqslant i \leqslant d}\left|X_{\mathfrak{u}, s}^{i}-Z_{s}^{i}\right|<\delta, \quad \max _{0 \leqslant i \leqslant d}\left|Z_{s}^{i}-\mathcal{X}_{\mathfrak{u}, s}^{i}\right|<\delta .
$$

Then

$$
\max _{1 \leqslant i \leqslant d}\left(\mathcal{X}_{\mathfrak{u}, s}^{i}-\mathcal{X}_{\mathfrak{u}, s}^{i-1}\right) \geqslant \mathfrak{b}
$$

It follows that for every $\gamma_{1}>\gamma / \sqrt{\mathfrak{u}}$ and every $t \in\left[t_{*}-\gamma_{1} \frac{\sigma}{\varepsilon} \sqrt{\ln \left(\frac{\sigma}{\varepsilon}\right)}, t_{*}\right]$

$$
\begin{aligned}
\mathbb{P}\left(\tau_{\mathcal{X}, \mathfrak{b}} \leqslant t\right) \geqslant & \mathbb{P}\left(\tau_{\mathfrak{u}, \mathfrak{b}+4 \delta} \leqslant t\right)-\mathbb{P}\left(\tau_{\mathfrak{u}, \mathfrak{b}} \leqslant t_{*}-\gamma_{1} \frac{\sigma}{\varepsilon} \sqrt{\ln \left(\frac{\sigma}{\varepsilon}\right)}\right) \\
& -\sum_{i=1}^{d-1} \mathbb{P}\left(\sup _{s \in\left[t_{*}-\gamma_{1} \frac{\sigma}{\varepsilon} \sqrt{\ln \left(\frac{\sigma}{\varepsilon}\right), t_{*}}\right]}\left|X_{\mathfrak{u}, s}^{i}-Z_{s}^{i}\right|>\delta\right)-\mathbb{P}\left(S_{t_{*}}^{*} \geqslant \delta\right) \\
= & \mathbb{P}\left(\tau_{\mathfrak{u}, \mathfrak{b}+4 \delta} \leqslant t\right)-o(1),
\end{aligned}
$$

where we used theorem 2, lemma 7 and proposition 8 . The rest of the proof goes along the same lines as in theorem 3 .

Upper bound. Assume that for some $s$ we have

$$
\max _{1 \leqslant i \leqslant d}\left(\mathcal{X}_{\mathfrak{u}, s}^{i}-\mathcal{X}_{\mathfrak{u}, s}^{i-1}\right) \geqslant \mathfrak{b}, \quad \max _{0 \leqslant i \leqslant d}\left|Z_{s}^{i}-\mathcal{X}_{\mathfrak{u}, s}^{i}\right|<\delta,
$$

Then

$$
\max _{1 \leqslant i \leqslant d}\left(Z_{s}^{i}-Z_{s}^{i-1}\right) \geqslant \mathfrak{b}-2 \delta .
$$

If, additionally

$$
\max _{0 \leqslant i \leqslant d}\left|X_{\mathfrak{u}, s}^{i}-Z_{s}^{i}\right|<\delta
$$

then

$$
\max _{1 \leqslant i \leqslant d}\left(X_{\mathfrak{u}, s}^{i}-X_{\mathfrak{u}, s}^{i-1}\right) \geqslant \mathfrak{b}-4 \delta .
$$

It follows that for every $t \in\left[t_{*}-\gamma_{1} \frac{\sigma}{\varepsilon} \sqrt{\ln \left(\frac{\sigma}{\varepsilon}\right)}, t_{*}\right]$

$$
\begin{aligned}
\mathbb{P}\left(\tau_{\mathcal{X}, \mathfrak{b}} \leqslant t\right)= & \mathbb{P}\left(\tau_{\mathcal{X}, \mathfrak{b}} \leqslant t_{*}-\gamma_{1} \frac{\sigma}{\varepsilon} \sqrt{\ln \left(\frac{\sigma}{\varepsilon}\right)}\right)+\mathbb{P}\left(\tau_{\mathcal{X}, \mathfrak{b}} \in\left[t_{*}-\gamma_{1} \frac{\sigma}{\varepsilon} \sqrt{\ln \left(\frac{\sigma}{\varepsilon}\right)}, t\right]\right) \\
\leqslant & \mathbb{P}\left(\tau_{Z, \mathfrak{b}-2 \delta} \leqslant t_{*}-\gamma_{1} \frac{\sigma}{\varepsilon} \sqrt{\ln \left(\frac{\sigma}{\varepsilon}\right)}\right)+\mathbb{P}\left(\tau_{\mathfrak{u}, \mathfrak{b}-4 \delta} \leqslant t\right) \\
& +\sum_{i=1}^{d-1} \mathbb{P}\left(\sup _{s \in\left[t_{*}-\gamma_{1} \frac{\sigma}{\varepsilon} \sqrt{\ln \left(\frac{\sigma}{\varepsilon}\right), t_{*}}\right]}\left|X_{\mathfrak{u}, t}^{i}-Z_{t}^{i}\right|>\delta\right)+\mathbb{P}\left(S_{t_{*}}^{*} \geqslant \delta\right) \\
= & \mathbb{P}\left(\tau_{\mathfrak{u}, \mathfrak{b}-4 \delta} \leqslant t\right)+o(1),
\end{aligned}
$$


where we used lemmas 6,7 and proposition 8 . The rest of the proof goes along the same lines as in theorem 3 .

\section{Auxiliary technical results}

\subsection{Some properties of the process $Z$}

7.1.1. Scalar analogues of $\boldsymbol{Z}$. We work under the assumptions (15) and P. Recall that under assumption $\mathrm{P}$ our potential $U$ is convex on $[1, \mathfrak{b}]$ and there exist finite positive constants $\kappa_{\min }, \kappa_{\max }$ and $K$ such that

$$
\begin{gathered}
\kappa_{\min } \leqslant U^{\prime \prime}(x) \leqslant \kappa_{\max }, \quad x \in[1, \mathfrak{b}], \\
\left|U^{\prime \prime \prime}(x)\right| \leqslant K, \quad x \in[1, \mathfrak{b}] .
\end{gathered}
$$

Lemma 9. Let $U:[1, \mathfrak{b}] \rightarrow \mathbb{R}$ be a function satisfying assumption $\mathrm{P}$. Set $\phi(t):=U^{\prime \prime}\left(1+\frac{\varepsilon t}{d}\right)$, $t \in\left[0, t_{*}\right]$ and $\mathfrak{u}:=\phi\left(t_{*}\right)=U^{\prime \prime}(\mathfrak{b})$, where $t_{*}:=d(\mathfrak{b}-1) / \varepsilon$.

Consider two scalar stochastic differential equations

$$
\mathrm{d} \mathfrak{Y}_{t}=-\mathfrak{u} \mathfrak{Y}_{t} \mathrm{~d} t+\sigma \mathrm{d} B_{t}, \quad t \geqslant 0, \quad \mathfrak{Y}_{0}=0,
$$

and

$$
\mathrm{d} \mathfrak{Z}_{t}=-\phi(t) \mathfrak{Z}_{t} \mathrm{~d} t+\sigma \mathrm{d} B_{t}, \quad t \geqslant 0, \quad \mathfrak{Z}_{0}=0 .
$$

Assume that $\varepsilon, \sigma \rightarrow 0$ with (15) being true. Let

$$
\mathcal{T}=\mathcal{T}\left(\sigma, \varepsilon, \gamma_{1}\right):=\left[t_{*}-\left(\gamma_{1} \sigma / \varepsilon\right) \sqrt{\ln (\sigma / \varepsilon)}, t_{*}\right]
$$

Then, for any $\gamma_{1}>0$ and $\theta>0$, we have

$$
\mathbb{P}\left(\sup _{t \in \mathcal{T}}\left|\mathfrak{Y}_{t}-\mathfrak{Z}_{t}\right|>\frac{\theta \sigma}{\sqrt{\ln (\sigma / \varepsilon)}}\right) \rightarrow 0 .
$$

Proof. We will use the abbreviations $h:=\varepsilon / \sigma \rightarrow 0$ and $\psi:=\ln \left(h^{-1}\right) \rightarrow \infty$.

Step 1: we derive formulas for the variances of $\mathfrak{Y}, \mathfrak{Z}$ and their covariances. Note that the explicit solution of (27) is given by

$$
\mathfrak{Z}_{t}=\sigma \exp (-\Phi(t)) \int_{0}^{t} \exp (\Phi(s)) \mathrm{d} B_{s}
$$

where $\Phi(t):=\int_{0}^{t} \phi(u) \mathrm{d} u$. Therefore, for the covariance we have

$$
\operatorname{cov}\left(\mathfrak{Z}_{t_{1}}, \mathfrak{Z}_{t_{2}}\right)=\sigma^{2} \int_{0}^{\min \left(t_{1}, t_{2}\right)} \exp \left(2 \Phi(s)-\Phi\left(t_{1}\right)-\Phi\left(t_{2}\right)\right) \mathrm{d} s .
$$

As a special case, for the variance we obtain

$$
\operatorname{var} \mathfrak{Z}_{t}=\sigma^{2} \int_{0}^{t} \exp (2(\Phi(s)-\Phi(t))) \mathrm{d} s .
$$


Replacing $\phi(t)$ by the constant $\mathfrak{u}$ in these formulas yields

$$
\begin{aligned}
\mathfrak{Y}_{t} & =\sigma \int_{0}^{t} \exp (\mathfrak{u}(s-t)) \mathrm{d} B_{s} ; \\
\operatorname{cov}\left(\mathfrak{Y}_{t_{1}}, \mathfrak{Y}_{t_{2}}\right) & =\sigma^{2} \int_{0}^{\min \left(t_{1}, t_{2}\right)} \mathrm{e}^{\mathfrak{u}\left(2 s-t_{1}-t_{2}\right)} \mathrm{d} s=\frac{\sigma^{2}}{2 \mathfrak{u}}\left(\mathrm{e}^{-\mathfrak{u}\left|t_{1}-t_{2}\right|}-\mathrm{e}^{-\mathfrak{u}\left(t_{1}+t_{2}\right)}\right) ; \\
\operatorname{var} \mathfrak{Y}_{t} & =\frac{\sigma^{2}}{2 \mathfrak{u}}\left(1-\mathrm{e}^{-2 \mathfrak{u} t}\right) .
\end{aligned}
$$

For the covariance between $\mathfrak{Y}$ and $\mathfrak{Z}$, one obtains from (28) and (30) that

$$
\operatorname{cov}\left(\mathfrak{Y}_{t}, \mathfrak{Z}_{t}\right)=\sigma^{2} \int_{0}^{t} \mathrm{e}^{\mathfrak{u}(s-t)+\Phi(s)-\Phi(t)} \mathrm{d} s .
$$

We shall show that for $t \in \mathcal{T}$

$$
\begin{aligned}
\operatorname{var} \mathfrak{Y}_{t} & =\frac{\sigma^{2}}{2 \mathfrak{u}}(1+O(\varepsilon|\ln \varepsilon|)), \\
\operatorname{var} \mathfrak{Z}_{t} & =\frac{\sigma^{2}}{2 \phi(t)}(1+O(\varepsilon|\ln \varepsilon|)), \\
\operatorname{cov}\left(\mathfrak{Y}_{t}, \mathfrak{Z}_{t}\right) & =\frac{\sigma^{2}}{\phi(t)+\mathfrak{u}}(1+O(\varepsilon|\ln \varepsilon|)), \\
\mathbb{E}\left[\left(\mathfrak{Y}_{t}-\mathfrak{Z}_{t}\right)^{2}\right] & =\sigma^{2}\left(O\left(\sigma^{2} \psi\right)+O(\varepsilon|\ln \varepsilon|)\right),
\end{aligned}
$$

and

$$
\mathbb{E}\left[\left(\mathfrak{Z}_{t_{2}}-\mathfrak{Z}_{t_{1}}\right)^{2}\right] \leqslant C \sigma^{2}\left|t_{2}-t_{1}\right|, \quad t_{1}, t_{2} \leqslant t_{*}, \quad\left|t_{2}-t_{1}\right| \leqslant 1 .
$$

Step 2: variance analysis: proof of (34) and (35).

Under assumption (15) we have $\mathcal{T} \subseteq\left[t_{*} / 2, t_{*}\right]$.

Relation (34) follows now directly from (32), as

$$
\mathrm{e}^{-2 \mathfrak{u} t} \leqslant \mathrm{e}^{-2 \mathfrak{u} t_{*} / 2}=\mathrm{e}^{-\mathfrak{u} d(\mathfrak{b}-1) \varepsilon^{-1}} \ll \varepsilon|\ln \varepsilon| \quad \text { for } t \in \mathcal{T} .
$$

Now we move to the proof of (35). Recall that under assumption $\mathrm{P}$ the coefficient function $\phi$ is bounded away from zero on $\left[0, t_{*}\right]$, namely $\phi(t) \geqslant \kappa_{\min }>0, t \in\left[0, t_{*}\right]$. This implies that for $0 \leqslant s \leqslant t$

$$
\Phi(t)-\Phi(s)=\int_{s}^{t} \phi(u) \mathrm{d} u \geqslant \kappa_{\min }(t-s) .
$$

Therefore, for all $0 \leqslant w \leqslant t$, we have

$$
\int_{0}^{t-w} \mathrm{e}^{2(\Phi(s)-\Phi(t))} \mathrm{d} s \leqslant \int_{0}^{t-w} \mathrm{e}^{2 \kappa_{\min }(s-t)} \mathrm{d} s \leqslant \int_{-\infty}^{t-w} \mathrm{e}^{2 \kappa_{\min }(s-t)} \mathrm{d} s=\left(2 \kappa_{\min }\right)^{-1} \mathrm{e}^{-2 \kappa_{\min } w} .
$$

We use this for $w:=\kappa_{\min }^{-1}|\ln \varepsilon|$ to get that

$$
\int_{0}^{t-w} \mathrm{e}^{2(\Phi(s)-\Phi(t))} \mathrm{d} s \leqslant\left(2 \kappa_{\min }\right)^{-1} \varepsilon^{2} \ll \varepsilon|\ln \varepsilon| .
$$


In the essential zone $s \in[t-w, t]$ we use the Taylor expansion of $\Phi$ :

$$
\begin{aligned}
\Phi(s)-\Phi(t) & =\Phi^{\prime}(t)(s-t)+\frac{1}{2} \Phi^{\prime \prime}(\tilde{s})(s-t)^{2} \\
& =\phi(t)(s-t)+\frac{1}{2} \phi^{\prime}(\tilde{s})(s-t)^{2} \\
& =\phi(t)(s-t)+\frac{\varepsilon}{2 d} U^{\prime \prime \prime}(\tilde{x})(s-t)^{2},
\end{aligned}
$$

with some $\tilde{s} \in[s, t]$ and $\tilde{x}:=1+\frac{\varepsilon \tilde{s}}{d} \in[1, \mathfrak{b}]$.

Recall that under assumption $\mathrm{P}$ we have $\left|U^{\prime \prime \prime}(\tilde{x})\right| \leqslant K$. Further, using that $|s-t| \leqslant w=$ $\kappa_{\text {min }}^{-1}|\ln \varepsilon|$, we obtain

$$
\Phi(s)-\Phi(t) \leqslant \phi(t)(s-t)+\frac{K}{2 d \kappa_{\min }} \varepsilon|\ln \varepsilon|(t-s) .
$$

It follows that

$$
\begin{aligned}
\int_{t-w}^{t} \exp (2(\Phi(s)-\Phi(t))) \mathrm{d} s & \leqslant \int_{t-w}^{t} \exp \left(2\left(\phi(t)-\frac{K}{2 d \kappa_{\min }} \varepsilon|\ln \varepsilon|\right)(s-t)\right) \mathrm{d} s \\
& \leqslant \int_{0}^{\infty} \exp \left(-2\left(\phi(t)-\frac{K}{2 d \kappa_{\min }} \varepsilon|\ln \varepsilon|\right) u\right) \mathrm{d} u \\
& =\frac{1}{2\left(\phi(t)-\frac{K}{2 d \kappa_{\min }} \varepsilon|\ln \varepsilon|\right)} \\
& \leqslant \frac{1}{2 \phi(t)}(1+O(\varepsilon|\ln \varepsilon|))
\end{aligned}
$$

having used again that $U^{\prime \prime}$ (and so $\phi$ ) is bounded away from zero.

Putting together the last relation with (39) and (29) shows the upper bound in (35).

For the lower bound in (35), we argue similarly. Again we set $w:=\kappa_{\min }^{-1}|\ln \varepsilon|$. Using the Taylor expansion of $\Phi$ from (40), we obtain for $s \in[t-w, t]$

$$
\Phi(s)-\Phi(t) \geqslant \phi(t)(s-t)-\frac{\varepsilon}{d} K(s-t)^{2} \geqslant\left[\phi(t)+\frac{\varepsilon}{2 d} K w\right](s-t) .
$$

Using this estimate, we get that

$$
\begin{aligned}
\int_{0}^{t} \mathrm{e}^{2(\Phi(s)-\Phi(t))} \mathrm{d} s & \geqslant \int_{t-w}^{t} \mathrm{e}^{-2(t-s)[\phi(t)+\varepsilon w K /(2 d)]} \mathrm{d} s \\
& =\int_{0}^{w} \mathrm{e}^{-2 u[\phi(t)+\varepsilon w K /(2 d)]} \mathrm{d} u \\
& =\frac{1}{2[\phi(t)+\varepsilon w K /(2 d)]} \cdot\left(1-\mathrm{e}^{-2 w[\phi(t)+\varepsilon w K /(2 d)]}\right) .
\end{aligned}
$$

The last term is lower bounded by

$$
\begin{aligned}
\frac{1}{2 \phi(t)}\left(1-\frac{\varepsilon w K}{2 d \phi(t)}\right) \cdot\left(1-\mathrm{e}^{-2 w \kappa_{\min }}\right) & \geqslant \frac{1}{2 \phi(t)}\left(1-\frac{\varepsilon|\ln \varepsilon| K}{2 d \kappa_{\min }^{2}}\right) \cdot\left(1-\varepsilon^{2}\right) \\
& =\frac{1}{2 \phi(t)}(1-O(\varepsilon|\ln \varepsilon|))
\end{aligned}
$$


This shows (recall (29)) that $\operatorname{var} \mathfrak{Z}_{t} \geqslant \frac{1}{2 \phi(t)}(1-O(\varepsilon|\ln \varepsilon|))$, as required.

Step 3: covariance analysis: proof of (36) and (37).

Relation (36) follows in the same way as we proved (35), because the integral in (33) has the same structure as the integral in (29), the only difference being that $\Phi(t)$ is replaced by $\Phi(t)+\mathfrak{u} t$ and all properties used in the above proof carry over.

We now show (37). Using the Taylor expansion of $\phi$, we get that for $t \in \mathcal{T}$,

$$
\phi(t)=U^{\prime \prime}\left(1+\frac{\varepsilon t}{d}\right)=\mathfrak{u}+U^{\prime \prime \prime}(\tilde{x}) \frac{\varepsilon}{d}\left(t-t_{*}\right),
$$

with some $\tilde{x} \in[1, \mathfrak{b}]$. Therefore, for $t \in \mathcal{T}$ we have

$$
|\phi(t)-\mathfrak{u}| \leqslant \frac{\left|U^{\prime \prime \prime}(\tilde{x})\right|}{d} \cdot \varepsilon\left|t-t_{*}\right| \leqslant C \varepsilon \gamma_{1} h^{-1} \sqrt{\psi} \sim C \gamma_{1} \sigma \sqrt{|\ln \varepsilon|} \rightarrow 0,
$$

by the assumption (15) and assumption P. Using this in the following computation, we get:

$$
\begin{aligned}
\mathbb{E}\left[\left(\mathfrak{Y}_{t}-\mathfrak{Z}_{t}\right)^{2}\right] & =\operatorname{var} \mathfrak{Y}_{t}+\operatorname{var} \mathfrak{Z}_{t}-2 \operatorname{cov}\left(\mathfrak{Y}_{t}, \mathfrak{Z}_{t}\right) \\
& =\sigma^{2}\left(\frac{1}{2 \mathfrak{u}}+\frac{1}{2 \phi(t)}-\frac{2}{\mathfrak{u}+\phi(t)}+O(\varepsilon|\ln \varepsilon|)\right) \\
& =\sigma^{2}\left(\frac{\phi(t) \mathfrak{u}+\phi(t)^{2}+\mathfrak{u}^{2}+\phi(t) \mathfrak{u}-4 \phi(t) \mathfrak{u}}{2 \mathfrak{u} \phi(t)(\mathfrak{u}+\phi(t))}+O(\varepsilon|\ln \varepsilon|)\right) \\
& =\sigma^{2}\left(\frac{(\phi(t)-\mathfrak{u})^{2}}{2 \mathfrak{u} \phi(t)(\mathfrak{u}+\phi(t))}+O(\varepsilon|\ln \varepsilon|)\right) \\
& =\sigma^{2}\left(O\left((\phi(t)-\mathfrak{u})^{2}\right)+O(\varepsilon|\ln \varepsilon|)\right) \\
& =\sigma^{2}\left(O\left(\sigma^{2} \psi\right)+O(\varepsilon|\ln \varepsilon|)\right),
\end{aligned}
$$

as stated.

Step 4: proof of (38).

Let $0 \leqslant t_{1} \leqslant t_{2} \leqslant t_{*}$. Then, using (28), we have

$$
\begin{aligned}
\mathbb{E}\left[\left(\mathfrak{Z}_{t_{2}}-\mathfrak{Z}_{t_{1}}\right)^{2}\right] & =\sigma^{2}\left(\mathrm{e}^{-\Phi\left(t_{2}\right)}-\mathrm{e}^{-\Phi\left(t_{1}\right)}\right)^{2} \int_{0}^{t_{1}} \mathrm{e}^{2 \Phi(s)} \mathrm{d} s+\sigma^{2} \mathrm{e}^{-2 \Phi\left(t_{2}\right)} \int_{t_{1}}^{t_{2}} \mathrm{e}^{2 \Phi(s)} \mathrm{d} s \\
& =\sigma^{2}\left(\mathrm{e}^{\Phi\left(t_{1}\right)-\Phi\left(t_{2}\right)}-1\right)^{2} \int_{0}^{t_{1}} \mathrm{e}^{2\left(\Phi(s)-\Phi\left(t_{1}\right)\right)} \mathrm{d} s+\sigma^{2} \int_{t_{1}}^{t_{2}} \mathrm{e}^{2\left(\Phi(s)-\Phi\left(t_{2}\right)\right)} \mathrm{d} s \\
& \leqslant \sigma^{2}\left(\Phi\left(t_{2}\right)-\Phi\left(t_{1}\right)\right)^{2} \int_{0}^{t_{1}} \mathrm{e}^{2\left(\Phi(s)-\Phi\left(t_{1}\right)\right)} \sigma^{2} \mathrm{~d} s+\sigma^{2} \int_{t_{1}}^{t_{2}} \mathrm{e}^{0} \mathrm{~d} s \\
& \leqslant \sigma^{2}\left(\int_{t_{1}}^{t_{2}} \phi(u) \mathrm{d} u\right)^{2} \int_{0}^{t_{1}} \mathrm{e}^{2 \kappa_{\min }\left(s-t_{1}\right)} \mathrm{d} s+\sigma^{2}\left(t_{2}-t_{1}\right) \\
& \leqslant \sigma^{2} \kappa_{\max }^{2}\left(t_{2}-t_{1}\right)^{2} \frac{1}{2 \kappa_{\min }}+\sigma^{2}\left(t_{2}-t_{1}\right) \leqslant C \sigma^{2}\left(t_{2}-t_{1}\right)
\end{aligned}
$$

if $t_{2}-t_{1} \leqslant 1$, as required in (38).

Step 5: probability evaluation. Define $\mathcal{D}_{t}:=\sigma^{-1}\left(\mathfrak{Y}_{t}-\mathfrak{Z}_{t}\right)$. The claim of the lemma is to show that

$$
\mathbb{P}\left(\sup _{t \in \mathcal{T}}\left|\mathcal{D}_{t}\right|>\frac{\theta}{\sqrt{\psi}}\right) \rightarrow 0
$$


Using the union bound and the fact that the length of $\mathcal{T}$ is $\gamma_{1} h^{-1} \sqrt{\psi}$, it is sufficient to show that

$$
h^{-1} \sqrt{\psi} \sup _{I \subseteq \mathcal{T},|I|=1} \mathbb{P}\left(\sup _{t \in I}\left|\mathcal{D}_{t}\right|>\frac{\theta}{\sqrt{\psi}}\right) \rightarrow 0 .
$$

In order to estimate the last probability, we will use the standard techniques from the theory of Gaussian processes, see $[13,14]$. Essentially, we have to estimate the maximal variance of $\left(\mathcal{D}_{t}\right)$ as well as the compactness properties of $I$ w.r.t. the distance induced by $\left(\mathcal{D}_{t}\right)$.

Fix $I \subseteq \mathcal{T}$ with $|I|=1$ and recall from (37) that

$$
V_{I}:=\max _{t \in I} \operatorname{var} \mathcal{D}_{t} \leqslant C\left(\sigma^{2} \psi+\varepsilon|\ln \varepsilon|\right) .
$$

Let $\bar{E}$ and $\bar{m}$ denote the expectation and the median of the random variable $\sup _{t \in I} \mathcal{D}_{t}$, respectively. It is known from the general Gaussian theory that $\bar{m} \leqslant \bar{E}$, see e.g. [13, lemma 12.2].

Assume that we have shown that

$$
\bar{E} \leqslant \frac{\theta}{2 \sqrt{\psi}}
$$

Then by concentration principle, see [13, theorem 12.2], we have

$$
\begin{aligned}
\mathbb{P}\left(\sup _{t \in I}\left|\mathcal{D}_{t}\right|>\frac{\theta}{\sqrt{\psi}}\right) & \leqslant 2 \mathbb{P}\left(\sup _{t \in I} \mathcal{D}_{t}>\frac{\theta}{\sqrt{\psi}}\right) \leqslant 2 \exp \left(-\frac{\left(\frac{\theta}{\sqrt{\psi}}-\bar{m}\right)^{2}}{2 V_{I}}\right) \\
& \leqslant 2 \exp \left(-\frac{\left(\frac{\theta}{\sqrt{\psi}}-\bar{E}\right)^{2}}{2 V_{I}}\right) \leqslant 2 \exp \left(-\frac{\theta^{2}}{8 \psi V_{I}}\right) \\
& \leqslant 2 \exp \left(-\frac{\theta^{2}}{8 \psi C\left(\sigma^{2} \psi+\varepsilon|\ln \varepsilon|\right)}\right) \\
& \leqslant 2 \exp \left(-\frac{\theta^{2}}{16 \psi C \max \left\{\sigma^{2} \psi, \varepsilon|\ln \varepsilon|\right\}}\right)
\end{aligned}
$$

which yields (41) because on the one hand (using (15))

$$
h^{-1} \sqrt{\psi} \mathrm{e}^{-c \psi^{-1} \varepsilon^{-1}|\ln \varepsilon|^{-1}} \ll \varepsilon^{-1} \sqrt{|\ln \varepsilon|} \mathrm{e}^{-c \varepsilon^{-1}|\ln \varepsilon|^{-2}} \rightarrow 0,
$$

while on the other hand-using that we can choose $\sigma^{2} \psi^{3}<\delta$ due to (15) - we obtain

$$
\begin{aligned}
h^{-1} \sqrt{\psi} \mathrm{e}^{-c \sigma^{-2} \psi^{-2}} & =h^{-1} \sqrt{\psi} \mathrm{e}^{-c \psi \sigma^{-2} \psi^{-3}} \\
& =h^{-1} \mathrm{e}^{-c \psi \sigma^{-2} \psi^{-3} / 2} \cdot \sqrt{\psi} \mathrm{e}^{-c \psi \sigma^{-2} \psi^{-3} / 2} \leqslant h^{-1} \mathrm{e}^{-c \psi /(2 \delta)} \cdot \sqrt{\psi} \mathrm{e}^{-c \psi /(2 \delta)} \\
& =h^{-1} h^{c /(2 \delta)} \cdot \sqrt{\psi} \mathrm{e}^{-c \psi /(2 \delta)} \rightarrow 0,
\end{aligned}
$$

for $\delta$ chosen small enough.

Step 6: we finally show (43).

We shall use the Dudley bound, see [13, theorem 14.1],

$$
\bar{E} \leqslant 4 \sqrt{2} \int_{0}^{\sqrt{\nabla_{I}}}(\ln N(\rho))^{1 / 2} \mathrm{~d} \rho,
$$


where $N(\rho)$ is the minimal number of $\rho$-balls that is needed to cover $I$ in the process-induced distance

$$
\Delta(s, t):=\mathbb{E}\left[\left|\mathcal{D}_{t}-\mathcal{D}_{s}\right|^{2}\right]^{1 / 2}
$$

From the result (38) (and the corresponding result for $\mathfrak{Y}$, which is simple to show) one obtains that

$$
\mathbb{E}\left[\left|\mathcal{D}_{t}-\mathcal{D}_{s}\right|^{2}\right]^{1 / 2} \leqslant C|t-s|^{1 / 2}, \quad t, s \in \mathcal{T}
$$

showing $N(\rho) \leqslant C^{\prime} \rho^{-2}$ for $\rho>0$. This implies that the Dudley integral in (44) is upper bounded by a constant times $\sqrt{V_{I}\left|\ln V_{I}\right|}$. The claim in (43) follows, if this quantity is of lower order compared to $\theta / \sqrt{\psi}$, i.e. we need to show that $V_{I}\left|\ln V_{I}\right| \ll \psi^{-1}$. Taking relation (42) into account, this is obtained from the following two relations:

$$
\begin{aligned}
& \psi^{-1} \gg \sigma^{2} \psi\left|\ln \left(\sigma^{2} \psi\right)\right|, \\
& \psi^{-1} \gg \varepsilon|\ln \varepsilon||\ln (\varepsilon|\ln \varepsilon|)| .
\end{aligned}
$$

Finally, using (15), we obtain

$$
\sigma^{2} \psi\left|\ln \left(\sigma^{2} \psi\right)\right| \ll\left(\sigma^{2} \psi\right)^{2 / 3}=\left(\sigma^{2} \psi^{3}\right)^{2 / 3} \psi^{-4 / 3} \ll \psi^{-4 / 3} \ll \psi^{-1},
$$

thus proving (45). Furthermore, from

$$
\psi=\ln (\sigma / \varepsilon) \leqslant|\ln \varepsilon|
$$

inequality (46) follows trivially.

7.1.2. A representation of $\boldsymbol{Z}$. Let us return to the SDE system (14) with a scalar function $\phi(\cdot)$, where $\phi(t)=U^{\prime \prime}\left(1+\frac{\varepsilon t}{d}\right)$. We will now connect (14) to the scalar processes treated in lemma 9.

The system (14) can be written in the vector form

$$
\left(Z_{t}^{1}, \ldots, Z_{t}^{d-1}\right)^{\top}=\phi(t) \mathcal{A}\left(Z_{t}^{1}, \ldots, Z_{t}^{d-1}\right)^{\top} \mathrm{d} t+\sigma \mathrm{d} \boldsymbol{B}_{t},
$$

where the $(d-1) \times(d-1)$-matrix $\mathcal{A}$ is defined by $\mathcal{A}_{i, i}=-2, \mathcal{A}_{i, i+1}=\mathcal{A}_{i+1, i}=-1$, and $\mathcal{A}_{i, j}=0$ otherwise ${ }^{5}$. Consider a diagonalization of $\mathcal{A}$ in the form $\mathcal{A}=Q^{\top} D Q$, where $D=\operatorname{diag}\left(\lambda_{1}, \ldots, \lambda_{d-1}\right)$ and $Q$ being a unitary operator. We only need that all eigenvalues $\lambda_{j}$, $1 \leqslant j \leqslant d-1$, are negative.

Further, consider the scalar SDEs:

$$
\mathrm{d} \mathfrak{Z}_{t}^{j}=\lambda_{j} \phi(t) \mathfrak{Z}_{t}^{j} \mathrm{~d} t+\sigma \mathrm{d} B_{t}^{j}, \quad t \geqslant 0, \quad \mathfrak{Z}_{0}^{j}=0, \quad j=1, \ldots, d,
$$

with independent Brownian motions $B^{j}$ and the same scalar function $\phi$ as above. Note that up to the prefactors $\lambda_{j}$, these are the processes treated in lemma 9 . The system of these equations can be rewritten in the vector form with $\mathfrak{Z}_{t}=\left(\mathfrak{Z}_{t}^{1}, \ldots, \mathfrak{Z}_{t}^{d-1}\right)$

$$
\mathrm{d} \mathfrak{Z}_{t}=D \mathfrak{Z}_{t} \phi(t) \mathrm{d} t+\sigma \mathrm{d} \boldsymbol{B}_{t}, \quad \mathfrak{Z}_{0}=0 .
$$

\footnotetext{
${ }^{5}$ Recall that $\boldsymbol{Z}$ has $d+1$ components, namely $\left(Z^{0}, \ldots, Z^{d}\right)$ with the trivial parts $Z^{0} \equiv 0$ and $Z_{t}^{d}=d+\varepsilon t$, while in the last equation we only want to represent the non-trivial components of $\boldsymbol{Z}$.
} 
Set

$$
g_{t}:=\left(g_{t}^{1}, \ldots, g_{t}^{d-1}\right):=-\int_{0}^{t} \exp \left(\mathcal{A}\left(\Phi_{t}-\Phi_{s}\right)\right) \mathrm{d} s \cdot \nu
$$

and further $g_{t}^{0} \equiv g_{t}^{d} \equiv 0$, where $\nu^{j}:=j / d, j=1, \ldots, d-1$, and $\Phi_{t}:=\int_{0}^{t} \phi(s) \mathrm{d} s$. It is simple to check that

$$
\mathcal{A} g_{t} \phi(t)=g_{t}^{\prime}+\nu
$$

This yields the following representation of $\left(Z_{t}^{1}, \ldots, Z_{t}^{d-1}\right)$ in terms of processes $\left(\mathfrak{Z}_{t}^{1}, \ldots, \mathfrak{Z}_{t}^{d-1}\right)$.

Lemma 10. Assume that the $\mathfrak{Z}$ solves (48). Then the following is a solution to (14):

$$
Z_{t}^{i}:=\frac{i}{d}(\varepsilon t+d)+\varepsilon g_{t}^{i}+\left(Q^{\top} \mathfrak{Z}\right)_{t}^{i}, \quad i=1, \ldots, d-1,
$$

where $\left(g_{t}^{i}\right)$ are bounded deterministic functions defined above.

Proof. Note that

$$
\begin{aligned}
\left(Z_{t}^{i+1}-\right. & \left.2 Z_{t}^{i}+Z_{t}^{i-1}\right) \phi(t) \mathrm{d} t+\sigma \mathrm{d} \tilde{B}_{t}^{i} \\
= & \frac{i+1-2 i+i-1}{d}(\varepsilon t+d) \phi(t) \mathrm{d} t+\varepsilon\left(g_{t}^{i+1}-2 g_{t}^{i}+g_{t}^{i-1}\right) \phi(t) \mathrm{d} t \\
& +\left(\left(Q^{\top} \mathfrak{Z}\right)_{t}^{i+1}-2\left(Q^{\top} \mathfrak{Z}\right)_{t}^{i}+\left(Q^{\top} \mathfrak{Z}\right)_{t}^{i-1}\right) \phi(t) \mathrm{d} t+\sigma \mathrm{d} \tilde{B}_{t}^{i} \\
= & \varepsilon\left(\mathcal{A} g_{t} \phi(t)\right)^{i} \mathrm{~d} t+\left(\mathcal{A} Q^{\top} \mathfrak{Z}_{t} \phi(t)\right)^{i} \mathrm{~d} t+\sigma \mathrm{d} \tilde{B}_{t}^{i} \\
= & \varepsilon\left(g_{t}^{\prime}+\nu\right)^{i} \mathrm{~d} t+\left(Q^{\top} D \mathfrak{Z}_{t} \phi(t)\right)^{i} \mathrm{~d} t+\sigma \mathrm{d}\left(Q^{\top} B\right)_{t}^{i} \\
= & \varepsilon\left(g_{t}^{\prime}+\boldsymbol{\nu}\right)^{i} \mathrm{~d} t+\left(Q^{\top} \mathfrak{Z}\right)_{t}^{i} \mathrm{~d} t \\
= & \mathrm{d} Z_{t}^{i},
\end{aligned}
$$

where we used (48) in the last but one step. Also the initial condition is verified:

$$
Z_{0}^{i}=i+\varepsilon g_{0}^{i}+\left(Q^{\top} \mathfrak{Z}\right)_{0}^{i}=i
$$

Let us finally show that the functions $\left(g_{t}^{i}\right)$ are bounded. Indeed, let $\mu:=\min \left|\lambda_{j}\right|>0$. Then

$$
\begin{aligned}
\left\|g_{t}\right\|_{\infty} & \leqslant\left\|g_{t}\right\|_{2} \\
& \leqslant \int_{0}^{t}\left\|\exp \left(\mathcal{A}\left(\Phi_{t}-\Phi_{s}\right)\right) \boldsymbol{\nu}\right\|_{2} \mathrm{~d} s \\
& =\int_{0}^{t}\left\|Q^{\top} \exp \left(D\left(\Phi_{t}-\Phi_{s}\right)\right) Q \boldsymbol{\nu}\right\|_{2} \mathrm{~d} s \\
& =\int_{0}^{t}\left\|\exp \left(D\left(\Phi_{t}-\Phi_{s}\right)\right) Q \boldsymbol{\nu}\right\|_{2} \mathrm{~d} s
\end{aligned}
$$




$$
\begin{aligned}
=\int_{0}^{t}\left(\sum_{j=1}^{d-1} \exp \left(-2 \lambda_{j}\left(\Phi_{t}-\Phi_{s}\right)\right)\left|(Q \boldsymbol{\nu})^{j}\right|^{2}\right)^{1 / 2} \mathrm{~d} s \\
\leqslant \int_{0}^{t}\left(\sum_{i=j}^{d-1} \exp \left(-2 \mu\left(\Phi_{t}-\Phi_{s}\right)\right)\left|(Q \nu)^{j}\right|^{2}\right)^{1 / 2} \mathrm{~d} s \\
=\int_{0}^{t} \exp \left(-\mu\left(\Phi_{t}-\Phi_{s}\right)\right) \cdot\|Q \boldsymbol{\nu}\|_{2} \mathrm{~d} s \\
=\int_{0}^{t} \exp \left(-\mu \int_{s}^{t} \phi(r) \mathrm{d} r\right) \cdot\|\boldsymbol{\nu}\|_{2} \mathrm{~d} s \\
\leqslant \int_{0}^{t} \exp \left(-\mu \int_{s}^{t} \kappa_{\min } \mathrm{d} r\right) \mathrm{d} s\|\boldsymbol{\nu}\|_{2} \\
=(1-\exp (-\mu t)) \frac{\|\boldsymbol{\nu}\|_{2}}{\mu \kappa_{\min }} .
\end{aligned}
$$

Remark 11. We proved in lemma 9 that the solution to (27) satisfies

$$
\begin{aligned}
\operatorname{var} \mathfrak{Z}_{t}^{j} & \leqslant C_{1} \sigma^{2}, \quad t \leqslant t_{*}, \\
\mathbb{E}\left[\left(\mathfrak{Z}_{t_{1}}^{j}-\mathfrak{Z}_{t_{2}}^{j}\right)^{2}\right] & \leqslant C_{2} \sigma^{2}\left|t_{1}-t_{2}\right|, \quad t_{1}, t_{2} \leqslant t_{*},\left|t_{1}-t_{2}\right| \leqslant 1,
\end{aligned}
$$

which carries over to linear combinations of the $\mathfrak{Z}^{j}$, e.g. to the processes $\left(Q^{\top} \mathfrak{Z}\right)^{i}$ in the representation (49).

\subsection{Proof of lemma 6}

Let us fix a break position $i \in\{1, \ldots, d\}$. Our starting point is a representation from lemma 10:

$$
Z_{t}^{i}-Z_{t}^{i-1}=q_{t}+\varepsilon\left(g_{t}^{i}-g_{t}^{i-1}\right)+\sum_{j=1}^{d-1} c_{i j} \mathfrak{J}_{t}^{j}=: q_{t}+\varepsilon \Delta_{t}^{i}+V_{t}^{i}, \quad i=0, \ldots, d-1,
$$

where $\Delta_{t}^{i}$ is a bounded deterministic part, and $\mathfrak{Z}^{j}$ are the independent processes from (47) (for this proof, independence is irrelevant). Let $D^{i}:=\sup _{t>0}\left|\Delta_{t}^{i}\right|$.

The exit condition of $i$ th component at a time $s$ is now equivalent to $q_{s}+\varepsilon \Delta_{t}^{i}+V_{s}^{i}=\mathfrak{b}$; in other words, $V_{s}^{i}=\frac{\varepsilon}{d}\left(t_{*}-s\right)-\varepsilon \Delta_{t}^{i}$. A necessary condition for the break is $V_{s}^{i} \geqslant \frac{\varepsilon}{d}\left(t_{*}-s\right)-$ $\varepsilon D^{i}$. We may restate it as

$$
\mathbb{P}\left(\tau_{Z, \mathfrak{b}}^{i} \leqslant t\right) \leqslant \mathbb{P}\left(\exists s \leqslant t: V_{s}^{i} \geqslant \frac{\varepsilon}{d}\left(t_{*}-s\right)-\varepsilon D^{i}\right) .
$$

By remark 11, the centred Gaussian process $V^{i}$, being a linear combination of the $\mathfrak{Z}^{j}$, inherits their following properties:

$$
\operatorname{var} V_{t}^{i} \leqslant C_{1} \sigma^{2}, \quad t \leqslant t_{*},
$$




$$
\mathbb{E}\left(V_{t_{2}}^{i}-V_{t_{1}}^{i}\right)^{2} \leqslant C_{2} \sigma^{2}\left|t_{1}-t_{2}\right|, \quad t_{1}, t_{2} \leqslant t_{*},\left|t_{1}-t_{2}\right| \leqslant 1,
$$

with the constants $C_{1}, C_{2}$ depending on dimension $d$ and potential $U$. By standard arguments of Gaussian process theory these bounds yield

$$
\mathbb{P}\left(\sup _{s \in[t-1, t]}\left|V_{s}^{i}\right| \geqslant \sigma R\right) \leqslant \exp \left(-C_{3} R^{2}\right), \quad R>0, \quad 1 \leqslant t \leqslant t_{*},
$$

with some $C_{3}=C_{3}\left(C_{1}, C_{2}\right)$.

By using (51), it follows that

$$
\begin{aligned}
\mathbb{P}\left(\tau_{Z, \mathfrak{b}}^{i} \leqslant t\right) \leqslant & \sum_{k=0}^{\infty} \mathbb{P}\left(\sup _{s \in[t-k-1, t-k]}\left|V_{s}^{i}\right| \geqslant \frac{\varepsilon}{d}\left(t_{*}-t+\left(k-d D^{i}\right)\right)\right) \\
\leqslant & \sum_{k=0}^{\infty} \exp \left(-C_{3}\left[\frac{\varepsilon}{d}\left(t_{*}-t+\left(k-d D^{i}\right)\right)\right]^{2} / \sigma^{2}\right) \\
\leqslant & \left.\exp \left(-C_{3}\left[\frac{\varepsilon}{d}\left(t_{*}-t\right)\right]^{2} / \sigma^{2}\right) \sum_{k=0}^{\infty} \exp \left(-2 C_{3} \frac{\varepsilon^{2}}{d^{2} \sigma^{2}}\left(t_{*}-t\right)\left(k-d D^{i}\right)\right)\right) \\
= & \exp \left(-C_{3}\left[\frac{\varepsilon}{d}\left(t_{*}-t\right)\right]^{2} / \sigma^{2}\right)\left[1-\exp \left(-2 C_{3} \frac{\varepsilon^{2}}{d^{2} \sigma^{2}}\left(t_{*}-t\right)\right)\right]^{-1} \\
& \left.\left.\times \exp \left(2 C_{3} \frac{\varepsilon^{2}}{d^{2} \sigma^{2}}\left(t_{*}-t\right) D^{i}\right)\right)\right) .
\end{aligned}
$$

Letting here $t=t_{*}-\gamma_{1} \frac{\sigma}{\varepsilon} \sqrt{\ln \left(\frac{\sigma}{\varepsilon}\right)}$, as in the assertion of lemma 6 , we obtain

$$
\begin{aligned}
\mathbb{P}\left(\tau_{Z, \mathfrak{b}}^{i} \leqslant t\right) & \leqslant\left(\frac{\sigma}{\varepsilon}\right)^{-C_{3} \gamma_{1}^{2} / d^{2}}\left[2 C_{3} \frac{\gamma_{1} \varepsilon}{d^{2} \sigma} \sqrt{\ln \left(\frac{\sigma}{\varepsilon}\right)}\right]^{-1}(1+o(1)) \\
& \leqslant\left(\frac{\sigma}{\varepsilon}\right)^{1-C_{3} \gamma_{1}^{2} / d^{2}} \frac{d^{2}}{2 C_{3} \gamma_{1}}\left(\ln \left(\frac{\sigma}{\varepsilon}\right)\right)^{-1 / 2}(1+o(1)) \rightarrow 0,
\end{aligned}
$$

if we choose $\gamma_{1}$ so large that $C_{3} \gamma_{1}^{2} / d^{2}>1$. This finishes the proof of lemma 6 .

\subsection{Proof of lemma 7}

As above, we set $\mathcal{T}:=\left[t_{*}-\gamma_{1} \frac{\sigma}{\varepsilon} \sqrt{\ln \sigma / \varepsilon}, t_{*}\right]$.

By lemma 10, the solutions to (10) and (14) can be represented by

$$
X_{\mathfrak{u}, t}^{i}=\frac{i}{d}(\varepsilon t+d)+\varepsilon g_{t}^{i}+\left(Q^{\top} \mathfrak{Y}\right)_{t}^{i}
$$

and

$$
Z_{t}^{i}=\frac{i}{d}(\varepsilon t+d)+\varepsilon \tilde{g}_{t}^{i}+\left(Q^{\top} \mathfrak{Z}\right)_{t}^{i},
$$

respectively, where $\boldsymbol{g}$ and $\tilde{\boldsymbol{g}}$ are bounded deterministic functions and $\mathfrak{Y}$ and $\mathfrak{Z}$ are the vectors of the solutions to (27) with constant prefactor $\mathfrak{u}$ and varying prefactor $\phi$, respectively. 
For the differences, we have

$$
X_{\mathfrak{u}, t}^{i}-Z_{t}^{i}=\varepsilon\left(g_{t}^{i}-\tilde{g}_{t}^{i}\right)+\left(Q^{\top}(\mathfrak{Y}-\mathfrak{Z})\right)_{t}^{i},
$$

so that (using the boundedness of $g_{t}$ and $\tilde{g}_{t}$ ) for some $K>0$ and all $t \geqslant 0$

$$
\begin{aligned}
\left\|\boldsymbol{X}_{\mathfrak{u}, t}-\boldsymbol{Z}_{t}\right\|_{\infty} & \leqslant \varepsilon K+\left\|Q^{\top}(\mathfrak{Y}-\mathfrak{Z})\right\|_{\infty} \\
& \leqslant \varepsilon K+\left\|Q^{\top}(\mathfrak{Y}-\mathfrak{Z})\right\|_{2} \\
& =\varepsilon K+\|\mathfrak{Y}-\mathfrak{Z}\|_{2} \\
& \leqslant \varepsilon K+\sqrt{d-1}\|\mathfrak{Y}-\mathfrak{Z}\|_{\infty} .
\end{aligned}
$$

Fix $\theta>0$. Using that $\varepsilon \ll \sigma / \sqrt{\ln \left(\frac{\sigma}{\varepsilon}\right)}$, we have, as $\varepsilon$, sigma $\rightarrow 0$,

$$
\begin{aligned}
\mathbb{P}\left(\sup _{t \in \mathcal{T}}\left\|\boldsymbol{X}_{\mathfrak{u}, t}-\boldsymbol{Z}_{t}\right\|_{\infty}>\frac{\theta \sigma}{\sqrt{\ln (\sigma \varepsilon)}}\right) & \leqslant \mathbb{P}\left(\sqrt{d-1} \sup _{t \in \mathcal{T}}\left\|\mathfrak{Y}_{t}-\mathfrak{Z}_{t}\right\|_{\infty}>\frac{1}{2} \frac{\theta \sigma}{\sqrt{\ln (\sigma \varepsilon)}}\right) \\
& \leqslant \sum_{i=1}^{d-1} \mathbb{P}\left(\sup _{t \in \mathcal{T}}\left|\mathfrak{Y}_{t}^{i}-\mathfrak{Z}_{t}^{i}\right|>\frac{(\theta /(2 \sqrt{d-1})) \sigma}{\sqrt{\ln (\sigma \varepsilon)}}\right) \rightarrow 0
\end{aligned}
$$

by lemma 9 . This finishes the proof of lemma 7.

\subsection{Proof of proposition 4}

Let, as above, $\mathcal{A}$ denote the discrete Laplace operator in one dimension with $d$ supporting points, i.e. the $(d-1)$-dimensional square matrix $\mathcal{A}$ with $\mathcal{A}_{i, j}=-2$ when $i=j, \mathcal{A}_{i, j}=1$ when $|i-j|=1$, and $\mathcal{A}_{i, j}=0$ otherwise. The largest eigenvalue $\lambda_{1}$ of $\mathcal{A}$ is strictly negative, namely $\lambda_{1}=-2(1-\cos (\pi / d))$.

By assumption $\mathrm{P}, U^{\prime \prime}$ is continuous and strictly positive on $[1, \mathfrak{b}]$. Therefore, there exist some $r>0$ and $u_{2}=u_{2}(r)>0$ such that $U^{\prime \prime}(x)>u_{2}(r)$ for all $x \in[1, \mathfrak{b}+r]$. By the continuity of the third derivative of $U$, we have $u_{3}(r):=\sup \left\{\left|U^{\prime \prime \prime}(x)\right|:|x| \leqslant \mathfrak{b}+r\right\}<\infty$. We define the constant

$$
c_{r}:=c(U, d, r):=\frac{6(d-1) u_{3}(r)}{\left|\lambda_{1}\right| u_{2}(r)}
$$

and the stopping time

$$
t_{r}(\omega):=\inf \left\{t \in \mathbb{R}: \exists i \leqslant n \quad \text { with }\left|\mathcal{X}_{s}^{i}(\omega)-\mathcal{X}_{s}^{i-1}(\omega)\right| \geqslant \mathfrak{b}+r\right\} \wedge t_{*} .
$$

Proposition 12. Let $r, c_{r}$ be as above. For all $\omega \in \Omega$ and all $t \leqslant t_{r}(\omega)$ we have

$$
S_{t}^{*}(\omega) \leqslant c_{r}\left(\left(S_{t}^{*}(\omega)\right)^{2}+\frac{1}{2}\left(M_{t}^{*}(\omega)\right)^{2}\right) .
$$

Proof. Let $t$ be arbitrary at first. We define $\mathcal{W}_{t}=\mathcal{X}_{t}-\boldsymbol{Z}_{t}$. Since $\boldsymbol{Z}$ and $\mathcal{X}$ are driven by the same Brownian motions, the process $\mathcal{W}$ fulfils

$$
\begin{aligned}
\mathrm{d} \mathcal{W}_{t}^{i}= & \mathrm{d} \mathcal{X}_{t}^{i}-\mathrm{d} Z_{t}^{i}=U^{\prime}\left(\mathcal{X}_{t}^{i+1}-\mathcal{X}_{t}^{i}\right) \mathrm{d} t-U^{\prime}\left(\mathcal{X}_{t}^{i}-\mathcal{X}_{t}^{i-1}\right) \mathrm{d} t \\
& -U^{\prime \prime}\left(q_{t}\right)\left(Z_{t}^{i+1}+Z_{t}^{-1}-2 Z_{t}^{i}\right) \mathrm{d} t .
\end{aligned}
$$


By adding and subtracting the term $U^{\prime \prime}\left(q_{t}\right)\left(\mathcal{X}_{t}^{i+1}+\mathcal{X}_{t}^{i-1}-2 \mathcal{X}_{t}^{i}\right) \mathrm{d} t$, and making the definition

$$
\left(\psi_{t}(x)\right)^{i}:=U^{\prime}\left(x^{i+1}-x^{i}\right)-U^{\prime}\left(x^{i}-x^{i-1}\right)-U^{\prime \prime}\left(q_{t}\right)\left(x^{i+1}+x^{i-1}-2 x^{i}\right),
$$

we obtain

$$
\mathrm{d} \mathcal{W}_{t}^{i}=\left(\psi_{t}\left(\mathcal{X}_{t}\right)\right)^{i}+U^{\prime \prime}\left(q_{t}\right)\left(\mathcal{W}_{t}^{i+1}-\mathcal{W}_{t}^{i-1}-2 \mathcal{W}_{t}^{i}\right) \mathrm{d} t
$$

In matrix notation, using the discrete Laplacian, this reads as

$$
\mathrm{d} \mathcal{W}_{t}=\boldsymbol{\psi}_{t}\left(\mathcal{X}_{t}\right)+\mathcal{U}^{\prime \prime}\left(q_{t}\right) \mathcal{A} \mathcal{W}_{t} \mathrm{~d} t
$$

and we get

$$
\mathcal{W}_{t}=\int_{0}^{t} \mathrm{e}^{\int_{s}^{t} U^{\prime \prime}\left(q_{v}\right) \mathrm{d} v \mathcal{A}} \boldsymbol{\psi}_{s}\left(\mathcal{X}_{s}\right) \mathrm{d} s
$$

The largest eigenvalue of the matrix $\mathrm{e}_{s}^{t} U^{\prime \prime}\left(q_{v}\right) \mathrm{d} v \mathcal{A}$ is bounded above by $\mathrm{e}^{(t-s) u_{2} \lambda_{1}}$, and thus by the matrix norm inequality we obtain

$$
\begin{aligned}
\left\|\boldsymbol{Z}_{t}(\omega)-\mathcal{X}_{t}(\omega)\right\|_{2} & =\left\|\mathcal{W}_{t}(\omega)\right\|_{2} \leqslant \int_{0}^{t} \mathrm{e}^{(t-s) u_{2} \lambda_{1}}\left\|\psi_{s}\left(\mathcal{X}_{s}(\omega)\right)\right\|_{2} \mathrm{~d} s \\
& \leqslant \frac{1}{u_{2} \lambda_{1}} \sup _{s \leqslant t}\left\|\boldsymbol{\psi}_{s}\left(\mathcal{X}_{s}(\omega)\right)\right\|_{2}
\end{aligned}
$$

for all $t>0$ and all $\omega \in \Omega$.

When we Taylor expand the first two terms on the right-hand side of (52) around the point $q_{t}$, the terms of order one cancel the third term there, and the second order remainder terms give the estimate

$$
\left|\left(\psi_{s}(x)\right)^{i}\right| \leqslant \frac{1}{2}\left|U^{\prime \prime \prime}\left(\xi_{+}\right)\left(x^{i+1}-x^{i}-q_{s}\right)^{2}\right|+\frac{1}{2}\left|U^{\prime \prime \prime}\left(\xi_{-}\right)\left(x^{i}-x^{i-1}-q_{s}\right)^{2}\right|,
$$

where $\xi_{+}$lies between $q_{s}$ and $x^{i+1}-x^{i}$, and where $\xi_{-}$lies between $q_{s}$ and $x^{i}-x^{i-1}$.

With $s \leqslant t_{r}(\omega)$ and $x^{i}=\mathcal{X}_{s}(\omega)$, the definition of $t_{r}(\omega)$ and the fact that $0 \leqslant q_{s} \leqslant \mathfrak{b}$ for $s \leqslant t \leqslant t_{0} \leqslant t_{*}$ yield $\left|\xi_{ \pm}\right|<\mathfrak{b}+r$. Therefore,

$$
\left|\psi_{s}\left(\mathcal{X}_{s}(\omega)\right)^{i}\right| \leqslant \frac{u_{3}(r)}{2}\left(\left(\mathcal{X}_{s}^{i+1}(\omega)-\mathcal{X}_{s}^{i}(\omega)-q_{s}\right)^{2}+\left(\mathcal{X}_{s}^{i}(\omega)-\mathcal{X}_{s}^{i-1}(\omega)-q_{s}\right)^{2}\right),
$$

and thus

$$
\left\|\psi_{s}\left(\mathcal{X}_{s}(\omega)\right)\right\|_{2} \leqslant(d-1) \sum_{i=1}^{d-1}\left|\psi_{s}\left(\mathcal{X}_{s}(\omega)\right)^{i}\right| \leqslant(d-1) u_{3}(r) \sum_{i=1}^{d}\left(\mathcal{X}_{s}^{i}(\omega)-\mathcal{X}_{s}^{i-1}(\omega)-q_{s}\right)^{2},
$$

for all $s \leqslant t_{r}(\omega)$. By the inequality

$$
\left(\mathcal{X}_{s}^{i}-\mathcal{X}_{s}^{i-1}-q_{s}\right)^{2} \leqslant 3\left(Z_{s}^{i}-\mathcal{X}_{s}^{i}\right)^{2}+3\left(Z_{s}^{i-1}-\mathcal{X}_{s}^{i-1}\right)^{2}+3\left(Z_{s}^{i}-Z_{s}^{i-1}-q_{s}\right)^{2},
$$

and since $Z^{0} \equiv \mathcal{X}^{0}$ and $Z^{d} \equiv \mathcal{X}^{d}$, we obtain

$$
\left\|\psi_{s}\left(\mathcal{X}_{s}(\omega)\right)\right\|_{2} \leqslant 6(d-1) u_{3}(r)\left(\left\|\mathcal{X}_{s}(\omega)-Z_{s}(\omega)\right\|_{2}^{2}+\frac{1}{2} \sum_{i=1}^{d}\left|Z_{s}^{i}-Z_{s}^{i-1}-q_{s}\right|^{2}\right) .
$$


Inserting this into the inequality (53), we obtain

$$
\left\|\boldsymbol{Z}_{\tilde{t}}-\mathcal{X}_{\tilde{t}}\right\|_{2} \leqslant \frac{6(d-1) u_{3}(r)}{u_{2}(r) \lambda_{1}}\left(\left(S_{\tilde{t}}^{*}(\omega)\right)^{2}+\frac{1}{2}\left(M_{\tilde{t}}^{*}(\omega)\right)^{2}\right)
$$

for all $\tilde{t} \leqslant t_{r}(\omega)$. The maps $\tilde{t} \mapsto\left(S_{\tilde{t}}^{*}(\omega)\right)$ and $\tilde{t} \mapsto\left(M_{\tilde{t}}^{*}(\omega)\right)$ are monotone increasing. Therefore for $t \leqslant t_{r}(\omega)$, we obtain the result by taking the supremum over $\tilde{t} \leqslant t$ on both sides of the above inequality.

Proof of proposition 4. We decompose

$$
\begin{aligned}
\mathbb{P}\left(S_{t}^{*} \geqslant R\right)= & \mathbb{P}\left(S_{t}^{*} \geqslant R, t \leqslant t_{r}, \sqrt{2} c_{r} M_{t}^{*}<1\right) \\
& +\mathbb{P}\left(S_{t}^{*} \geqslant R, t>t_{r}, \sqrt{2} c_{r} M_{t}^{*}<1\right) \\
& +\mathbb{P}\left(S_{t}^{*} \geqslant R, \sqrt{2} c_{r} M_{t}^{*} \geqslant 1\right) .
\end{aligned}
$$

The term (56) is simply estimated by $\mathbb{P}\left(\sqrt{2} c_{r} M_{t}^{*} \geqslant 1\right)$, giving the third term on the right-hand side of the claim.

Turning to the term (54), we will show that for all $\omega \in \Omega$ with $S_{t}(\omega) \geqslant R, t \leqslant t_{r}(\omega)$ and $\sqrt{2} c_{r} M_{t}^{*}(\omega)<1$, the inequality

$$
M_{t}^{*}(\omega)^{2} \geqslant \frac{S_{t}^{*}(\omega)}{2 c_{r}} \geqslant \frac{R}{2 c_{r}}
$$

holds, which then gives the first term on the right-hand side of the claim. To see (57), recall first that by proposition 12 we have

$$
c_{r}\left(S_{s}^{*}(\omega)\right)^{2}-S_{s}^{*}(\omega)+\frac{1}{2} c_{r}\left(M_{s}^{*}(\omega)\right)^{2} \geqslant 0
$$

for all $s \leqslant t_{r}(\omega)$. Since $t \leqslant t_{r}(\omega)$, this inequality holds for all $s \leqslant t$. Let $M>0$. The equation $y=c_{r}\left(y^{2}+M^{2} / 2\right)$ has two nonnegative solutions if and only if $c_{r}^{2} M^{2}<1 / 2$, and in this case the smaller one of those is given by

$$
\frac{1-\sqrt{1-2 c_{r}^{2} M^{2}}}{2 c_{r}}<2 c_{r} M^{2}
$$

By the condition $\sqrt{2} c_{r} M_{t}^{*}(\omega)<1$ and monotonicity we have $\sqrt{2} c_{r} M_{s}^{*}(\omega)<1$ for all $s \leqslant t$; by the above considerations (with $M=M_{s}^{*}(\omega)$ ), we find that for all $s \leqslant t$ and all $\omega$ fulfiling the relevant conditions in (54), the value of $S_{s}^{*}(\omega)$ can not be in the interval between the two solutions of the quadratic equation for any $s \leqslant t$. Since the function $s \mapsto S_{s}^{*}(\omega)$ is continuous and has the value 0 for $s=0$, it therefore has to stay to the left of the smaller root, and is therefore for $s=t$ bounded by $2 c_{r} M_{t}^{*}(\omega)^{2}$. We thus arrive at (57).

Finally we discuss the term (55). The considerations of the previous paragraph still apply, but only up to $s=t_{r}(\omega)<t$. Therefore while we do not have (57), we still know that

$$
S_{t_{r}(\omega)}^{*}(\omega) \leqslant 2 c_{r} M_{t_{r}(\omega)}^{*}(\omega)
$$

for all $\omega$ relevant to (55). Since $t_{r}(\omega)<t \leqslant t_{*}$, by definition of $t_{r}(\omega)$, there is at least one $i \leqslant d$ with $\left|\mathcal{X}_{t_{r}(\omega)}^{i}(\omega)-\mathcal{X}_{t_{r}(\omega)}^{i-1}(\omega)\right|=\mathfrak{b}+r$, and we get 


$$
\begin{aligned}
\mathfrak{b}+r= & \left|\mathcal{X}_{t_{r}(\omega)}^{i}(\omega)-\mathcal{X}_{t_{r}(\omega)}^{i-1}(\omega)\right| \leqslant\left|\mathcal{X}_{t_{r}(\omega)}^{i}(\omega)-Z_{t_{r}(\omega)}^{i}(\omega)\right| \\
& +\left|Z_{t_{r}(\omega)}^{i}(\omega)-Z_{t_{r}(\omega)}^{i-1}(\omega)\right|+\left|\mathcal{X}_{t_{r}(\omega)}^{i-1}(\omega)-Z_{t_{r}(\omega)}^{i-1}(\omega)\right| \\
\leqslant & \left|Z_{t_{r}(\omega)}^{i}(\omega)-Z_{t_{r}(\omega)}^{i-1}(\omega)\right|+2 S_{t_{r}(\omega)}^{*}(\omega) \\
\leqslant & \left|Z_{t_{r}(\omega)}^{i}(\omega)-Z_{t_{r}(\omega)}^{i-1}(\omega)\right|+4 c_{r}\left(M_{t_{r}(\omega)}^{*}(\omega)\right)^{2} .
\end{aligned}
$$

Therefore,

$$
\begin{aligned}
M_{t}^{*}(\omega) & \geqslant\left|Z_{t_{r}(\omega)}^{i}(\omega)-Z_{t_{r}(\omega)}^{i-1}(\omega)-q_{t_{r}(\omega)}\right| \geqslant\left|Z_{t_{r}(\omega)}^{i}(\omega)-Z_{t_{r}(\omega)}^{i-1}(\omega)\right|-\mathfrak{b} \\
& \geqslant r-4 c_{r}\left(M_{t_{r}(\omega)}^{*}(\omega)\right)^{2} \geqslant r-4 c_{r}\left(M_{t}^{*}(\omega)\right)^{2} .
\end{aligned}
$$

Therefore, $M_{t}^{*}(\omega)+4 c_{r}\left(M_{t}^{*}\right)(\omega)^{2} \geqslant r$ for all $\omega$ relevant to (55), and we obtain the second term on the right-hand side of the claim.

\subsection{Proof of proposition 8}

By proposition 4, it is enough to prove that for $t=t_{*}=\frac{d(\mathfrak{b}-1)}{\varepsilon}$ the right-hand side of (18) tends to zero, as $\varepsilon, \sigma \rightarrow 0$.

Under assumption (4) we have $\delta \rightarrow 0$. Therefore, the first probability in the right-hand side of (18) dominates two others. It remains to prove that for every $\theta>0$ we have

$$
\lim _{\varepsilon, \sigma \rightarrow 0} \mathbb{P}\left(M_{t_{*}}^{*} \geqslant \sqrt{\delta} / C\right)=0 .
$$

Since $\theta>0$ is arbitrary, we may drop $C$ here. Furthermore, by using the union bound, it is sufficient to prove that

$$
\lim _{\varepsilon, \sigma \rightarrow 0} \mathbb{P}\left(\sup _{0 \leqslant s \leqslant t_{*}}\left|Z_{s}^{i}-Z_{s}^{i-1}-q_{s}\right| \geqslant \sqrt{\delta}\right)=0, \quad i=1, \ldots, d .
$$

We fix $i$. By representation (50) we have

$$
\begin{aligned}
\mathbb{P}\left(\sup _{0 \leqslant s \leqslant t_{*}}\left|Z_{s}^{i}-Z_{s}^{i-1}-q_{s}\right| \geqslant \sqrt{\delta}\right) & \leqslant \mathbb{P}\left(\sup _{0 \leqslant s \leqslant t_{*}}\left|V_{s}^{i}\right| \geqslant \sqrt{\delta}-\varepsilon D_{i}\right) \\
& \leqslant\left(t_{*}+1\right) \max _{\left.0 \leqslant k \leqslant t_{*}\right]} \mathbb{P}\left(\sup _{k \leqslant s \leqslant k+1}\left|V_{s}^{i}\right| \geqslant \sqrt{\delta}-\varepsilon D_{i}\right) .
\end{aligned}
$$

Under (4) we have

$$
\frac{\varepsilon}{\delta}=\frac{\varepsilon}{\theta \sigma} \sqrt{\ln \left(\frac{\sigma}{\varepsilon}\right)} \rightarrow 0
$$

Hence, $\varepsilon \ll \delta \ll \sqrt{\delta}$, and eventually $\sqrt{\delta}-\varepsilon D_{i} \geqslant \sqrt{\delta} / 2$. By using (51), we obtain

$$
\mathbb{P}\left(\sup _{0 \leqslant s \leqslant t_{*}}\left|Z_{s}^{i}-Z_{s}^{i-1}-q_{s}\right| \geqslant \sqrt{\delta}\right) \leqslant\left(t_{*}+1\right) \exp \left\{-\frac{C_{3} \delta}{4 \sigma^{2}}\right\} .
$$

Notice that $t_{*} \approx \varepsilon^{-1}$, while under assumption (4) 


$$
\sigma^{-2} \delta=\theta \sigma^{-1} / \sqrt{\ln \left(\frac{\sigma}{\varepsilon}\right)} \geqslant \theta \sigma^{-1} / \sqrt{|\ln \varepsilon|}=\theta\left(\sigma^{2}|\ln \varepsilon|^{3}\right)^{-1 / 2}|\ln \varepsilon| \gg|\ln \varepsilon|,
$$

which completes the proof of proposition 8 .

\section{Acknowledgments}

This research was supported by the co-ordinated Grants of DFG (AU370/7) and RFBR (2051-12004).

\section{Data availability statement}

No new data were created or analysed in this study.

\section{ORCID iDs}

Frank Aurzada (D) https://orcid.org/0000-0002-4465-0297

Volker Betz (D) https://orcid.org/0000-0002-3538-7247

\section{References}

[1] Allman M and Betz V 2009 Breaking the chain Stoch. Proc. Appl. 119 2645-59

[2] Allman M, Betz V and Hairer M 2011 A chain of interacting particles under strain Stoch. Proc. Appl. 121 2014-42

[3] Aurzada F, Betz V and Lifshits M 2021 Breaking a chain of interacting Brownian particles Ann. Appl. Probab. (arXiv:1912.05168) (unpublished)

[4] Aurzada F, Betz V and Lifshits M 2021 Breaking a chain of interacting Brownian particles: a Gumbel limit theorem Teor. Veroyatnost. i Primenen. 66 231-60 (English version unpublished in Theory Probab. Appl.)

[5] Bao H, Huang Y, Yang Z, Sun Y, Bai Y, Miao Y, Chu P K, Xu K and Ma F 2018 Molecular dynamics simulation of nanocrack propagation in single-layer $\mathrm{MoS}_{2}$ nanosheets J. Phys. Chem. C 122 1351-60

[6] Berglund N and Gentz B 2006 Noise-Induced Phenomena in Slow-Fast Dynamical Systems (Series Probability and Its Applications) (Berlin: Springer)

[7] Diehl J, Gubinelli M and Perkowski N 2017 The Kardar-Parisi-Zhang equation as scaling limit of weakly asymmetric interacting Brownian motions Commun. Math. Phys. 354 549-89

[8] Doerr T P and Taylor P L 1994 Breaking in polymer chains. I. The harmonic chain J. Chem. Phys. 10110107

[9] Fugmann S and Sokolov I M 2009a Scaling of the rupture dynamics of polymer chains pulled at one end at a constant rate Phys. Rev. E 79021803

[10] Fugmann S and Sokolov I M 2009b Non-monotonic dependence of the polymer rupture force on molecule chain length Europhys. Lett. 8628001

[11] Funaki T and Spohn H 1997 Motion by mean curvature from the Ginzburg-Landau interface model Commun. Math. Phys. 185 1-36

[12] Lee C F 2009 Thermal breakage of a discrete one-dimensional string Phys. Rev. E 80031134

[13] Lifshits M A 1995 Gaussian Random Functions (Series Mathematics and Its Applications vol 322) (Dordrecht: Kluwer)

[14] Lifshits M 2012 Lectures on Gaussian Processes (Springer Briefs in Mathematics) (Heidelberg: Springer)

[15] Malyshev V A and Muzychka S A 2014 Dynamical phase transition in the simplest molecular chain model Theor. Math. Phys. 179 490-9 
[16] Malyshev V 2006 One-dimensional mechanical networks and crystals Moscow Math. J. 6 353-8

[17] Muzychka S A 2013 Mean exit time for a chain of $N=2,3,4$ oscillators Moscow Univ. Math. Bull. $68206-10$

[18] Patil S P and Heider Y 2019 A review on brittle fracture nanomechanics by all-atom simulations Nanomaterials 91050

[19] Razbin M, Benetatos P and Moosavi-Movahedi A A 2019 A first-passage approach to the thermal breakage of a discrete one-dimensional chain Soft Matter 152469

[20] Spohn H 1986 Equilibrium fluctuations for interacting Brownian particles Commun. Math. Phys. $1031-33$

[21] Varadhan S R S 1990 Scaling limits for interacting diffusions Commun. Math. Phys. 135 313-53 